University of Louisville

ThinkIR: The University of Louisville's Institutional Repository

Faculty Scholarship

$11-1-2020$

\title{
Galaxy and mass assembly: Luminosity and stellar mass functions in GAMA groups
}

\author{
J. A. Vazquez-Mata \\ University of Sussex \\ J. Loveday \\ University of Sussex \\ S. D. Riggs \\ University of Sussex \\ I. K. Baldry \\ Liverpool John Moores University \\ L. J.M. Davies \\ The University of Western Australia \\ See next page for additional authors
}

Follow this and additional works at: https://ir.library.louisville.edu/faculty

Part of the Astrophysics and Astronomy Commons

\section{ThinkIR Citation}

Vazquez-Mata, J. A.; Loveday, J.; Riggs, S. D.; Baldry, I. K.; Davies, L. J.M.; Robotham, A. S.G.; Holwerda, Benne W.; Brown, M. J.I.; Cluver, M. E.; Wang, L.; Alpaslan, M.; Bland-Hawthorn, J.; Brough, S.; Driver, S. P.; Hopkins, A. M.; Taylor, E. N.; and Wright, A. H., "Galaxy and mass assembly: Luminosity and stellar mass functions in GAMA groups" (2020). Faculty Scholarship. 471.

https://ir.library.louisville.edu/faculty/471

This Article is brought to you for free and open access by ThinkIR: The University of Louisville's Institutional Repository. It has been accepted for inclusion in Faculty Scholarship by an authorized administrator of ThinkIR: The University of Louisville's Institutional Repository. For more information, please contact thinkir@louisville.edu. 


\section{Authors}

J. A. Vazquez-Mata, J. Loveday, S. D. Riggs, I. K. Baldry, L. J.M. Davies, A. S.G. Robotham, Benne W.

Holwerda, M. J.I. Brown, M. E. Cluver, L. Wang, M. Alpaslan, J. Bland-Hawthorn, S. Brough, S. P. Driver, A.

M. Hopkins, E. N. Taylor, and A. H. Wright

This article is available at ThinkIR: The University of Louisville's Institutional Repository: https://ir.library.louisville.edu/ 


\title{
Galaxy and Mass Assembly: luminosity and stellar mass functions in GAMA groups
}

\author{
J.A. Vázquez-Mata, ${ }^{1,2}$ J. Loveday, ${ }^{1 \star}$ S.D. Riggs, ${ }^{1}$ I.K. Baldry, ${ }^{3}$ L.J.M. Davies,${ }^{4}$ \\ A.S.G. Robotham, ${ }^{4}$ B.W. Holwerda, ${ }^{5}$ M.J.I. Brown, ${ }^{6}$ M. E. Cluver, ${ }^{7,8}$ L. Wang, ${ }^{9,10}$ \\ M. Alpaslan, ${ }^{11}$ J. Bland-Hawthorn, ${ }^{12}$ S. Brough, ${ }^{13}$ S.P. Driver, ${ }^{4,14}$ A.M. Hopkins, ${ }^{15}$ \\ E.N. Taylor, ${ }^{7}$ A.H. Wright ${ }^{16,17}$ \\ ${ }^{1}$ Astronomy Centre, University of Sussex, Falmer, Brighton BN1 9QH, UK \\ ${ }^{2}$ Institute of Astronomy, National Autonomous University of Mexico, Mexico 04510 \\ ${ }^{3}$ Astrophysics Research Institute, Liverpool John Moores University, IC2, Liverpool Science Park, 146 Brownlow Hill, Liverpool, L3 5RF, UK \\ ${ }^{4}$ International Centre for Radio Astronomy Research (ICRAR), The University of Western Australia, 35 Stirling Highway, Crawley, WA6009, Australia \\ ${ }^{5}$ Department of Physics and Astronomy, University of Louisville, Louisville, KY 40292, USA \\ ${ }^{6}$ School of Physics, Monash University, Clayton, Victoria 3800, Australia \\ ${ }^{7}$ Centre for Astrophysics \& Supercomputing, Swinburne University of Technology, Hawthorn, VIC 3122, Australia \\ ${ }^{8}$ Department of Physics and Astronomy, University of the Western Cape, Robert Sobukwe Road, Bellville, 7535, South Africa \\ ${ }^{9}$ SRON Netherlands Institute for Space Research, Landleven 12, 9747 AD, Groningen, The Netherlands \\ ${ }^{10}$ Kapteyn Astronomical Institute, University of Groningen, Postbus 800, $9700 \mathrm{AV}$, Groningen, The Netherlands \\ ${ }^{11}$ Center for Cosmology and Particle Physics, Department of Physics, New York University, 726 Broadway, New York, NY 10003, USA \\ ${ }^{12}$ Sydney Institute for Astronomy, School of Physics, University of Sydney, NSW 2006, Australia \\ ${ }^{13}$ School of Physics, University of New South Wales, NSW 2052, Australia \\ ${ }^{14}$ School of Physics \& Astronomy, University of St Andrews, North Haugh, St Andrews, KY16 9SS, UK \\ ${ }^{15}$ Australian Astronomical Optics, Macquarie University 105 Delhi Rd, North Ryde, NSW 2113, Australia \\ ${ }^{16}$ Argelander-Institut für Astronomie, Universität Bonn, Auf dem Hügel 71, 53121 Bonn, Germany \\ ${ }^{17}$ Astronomisches Institut, Ruhr-Universität Bochum, Universitätsstr. 150, 44801 Bochum, Germany
}

Accepted XXX. Received YYY; in original form ZZZ

\begin{abstract}
How do galaxy properties (such as stellar mass, luminosity, star formation rate, and morphology) and their evolution depend on the mass of their host dark matter halo? Using the Galaxy and Mass Assembly (GAMA) group catalogue, we address this question by exploring the dependence on host halo mass of the luminosity function (LF) and stellar mass function (SMF) for grouped galaxies subdivided by colour, morphology and central/satellite. We find that spheroidal galaxies in particular dominate the bright and massive ends of the LF and SMF, respectively. More massive haloes host more massive and more luminous central galaxies. The satellite LF and SMF respectively show a systematic brightening of characteristic magnitude, and increase in characteristic mass, with increasing halo mass. In contrast to some previous results, the faint-end and low-mass slopes show little systematic dependence on halo mass. Semi-analytic models and simulations show similar or enhanced dependence of central mass and luminosity on halo mass. Faint and low-mass simulated satellite galaxies are remarkably independent of halo mass, but the most massive satellites are more common in more massive groups. In the first investigation of low-redshift LF and SMF evolution in group environments, we find that the red/blue ratio of galaxies in groups has increased since redshift $z \approx 0.3$ relative to the field population. This observation strongly suggests that quenching of star formation in galaxies as they are accreted into galaxy groups is a significant and ongoing process.
\end{abstract}

Key words: galaxies: groups: general — galaxies: luminosity function, mass function galaxies: evolution 
haloes (e.g. Press \& Schechter 1974; White \& Rees 1978). These haloes also attract baryons, a small fraction of which will condense into stars and thence form galaxies. How do galaxy properties, such as stellar mass, luminosity, star-formation rate, and morphology, depend on host halo mass and evolutionary history? The connection between galaxies and their host DM haloes is an active area of astrophysical research (see Wechsler \& Tinker 2018, for a recent review). One approach to studying this connection is to identify and weigh individual haloes using galaxies as tracers. Galaxy group catalogues provide a way to estimate the total mass of individual haloes down to $\sim 10^{12} \mathcal{M}_{\odot}$ via the (assumed virialized) galaxy motions within them (Eke et al. 2006; Robotham et al. 2011), or by weak-lensing calibrated scaling relations (Han et al. 2015; Viola et al. 2015).

The galaxy luminosity function (LF) and stellar mass function (SMF) are fundamental observables, giving a description of the population of galaxies in different environments, and contain valuable information about the physical processes that feature prominently in galaxy formation and evolution. The LF and SMF and their evolution provide important constraints on theories and models of galaxy formation and evolution (e.g. Benson et al. 2003; GonzalezPerez et al. 2014; Lacey et al. 2016; Lagos et al. 2018).

In the last few years, many authors have investigated the effect of environment on the LF, focusing on the dependence of the LF on the density contrast within spheres of different radii (e.g. Croton et al. 2005; Hoyle et al. 2005; Xia et al. 2006; Park et al. 2007; Phleps et al. 2007; McNaught-Roberts et al. 2014). These works agree that the LF varies significantly with environment, with characteristic magnitude brightening systematically with increasing local density. What is less clear is any systematic dependence of the faint-end slope with density, with some authors (e.g. Xia et al. 2006) claiming a steepening slope (i.e. more dwarf galaxies) in higher-density environments, while others (e.g. Croton et al. 2005; Hoyle et al. 2005; McNaught-Roberts et al. 2014) see little correlation. The SMF as a function of projected density has been presented by Peng et al. (2010), who find that the low-mass SMF of red galaxies is slightly steeper in the highest density quartile, while the low-mass slopes for blue galaxies are indistinguishable. While Mortlock et al. (2015, fig. 14) find a steeper SMF slope in high-density environments at redshifts $z \gtrsim 0.5$, they find the opposite in their low-redshift bin. Earlier, Baldry et al. (2006) found that characteristic mass increases with projected density.

Large spectroscopic surveys of galaxies, such as the Sloan Digital Sky Survey (SDSS; York et al. 2000) and the 2dF Galaxy Redshift Survey (2dFGRS; Colless et al. 2001) provide the potential for group-finding based on the redshift-space distribution of galaxies. Many authors have taken advantage of these surveys to construct galaxy group catalogues to explore multiple aspects of these systems, (e.g. Merchán \& Zandivarez 2002, 2005; Eke et al. 2004a; Yang et al. 2005, 2007; Berlind et al. 2006; Weinmann et al. 2006; Muñoz-Cuartas \& Müller 2012). In particular, the dependence of the galaxy LF on group environment has been investigated by, e.g. Eke et al. (2004b); Robotham et al. (2006, 2010); Zandivarez et al. (2006); Zandivarez \& Martínez (2011); Guo et al. (2014). These works mainly explored the variation of the Schechter (1976) function parameters, the characteristic magnitude $M^{*}$ and the faint-end slope $\alpha$, for different galaxy populations, as a function of the galaxy group virial mass, multiplicity, velocity dispersion, etc. Their results showed clear variations of $M^{*}$ and $\alpha$ with the different group properties. Robotham et al. (2010) found clear trends for steepening faint-end slope $\alpha$ as group mass and/or multiplicity increase for early-type galaxies, while a much suppressed relation was observed for the late-type population. Zandivarez \& Martínez (2011) found similar results.

Rather than measuring the number density of galaxies per unit volume, one can instead measure the average number of galaxies per host group (e.g. Yang et al. 2003). The conditional luminosity function (CLF), $\phi_{C}\left(L \mid \mathcal{M}_{h}\right)$, describes the average number of galaxies as a function of luminosity $L$ in groups of mass $\mathcal{M}_{h}$, i.e. average number per group rather than per unit volume, and can be considered an extension of the halo occupation distribution (HOD) model (e.g. Berlind \& Weinberg 2002; Brown et al. 2008). Similarly, the conditional stellar mass function (CSMF), $\phi_{C}\left(\mathcal{M}_{*} \mid \mathcal{M}_{h}\right)$, describes the average number of galaxies per group as a function of their stellar mass $\mathcal{M}_{*}$. Using the SDSS DR4 catalogue, Yang et al. $(2008,2009)$ found that the characteristic luminosity gets brighter, the characteristic mass increases, and the faint- and lowmass slopes of the CLF and CSMF get steeper, as halo mass increases. There is a danger, however, in characterising LF dependence on environment purely in terms of Schechter function parameters. The Schechter parameters $\left(\alpha, M^{*}\right)$ are strongly correlated, and also very sensitive to the limiting magnitude used in the fit (Croton et al. 2005, appendix C). Thus the Schechter function parametrization should only be used if (i) the fit is performed over a consistent magnitude range, and (ii) the functional fit is a good one (as confirmed by a $\chi^{2}$-test or likelihood ratio comparison with a non-parametric estimate).

The Galaxy and Mass Assembly (GAMA; Driver et al. 2009, 2011; Liske et al. 2015) survey provides an opportunity to reassess the galaxy LF and SMF dependence on host group properties. Although of smaller area than SDSS, GAMA provides spectroscopic redshifts two magnitudes fainter than SDSS, and, even more importantly for group studies, is highly complete, even in high-density group environments. The dependence of the galaxy LF on local environment, as defined by galaxy counts in $8 h^{-1} \mathrm{Mpc}$ spheres, has previously been presented for GAMA data by McNaught-Roberts et al. (2014), who found that denser environments contain redder and brighter galaxies than low-density environments. Alpaslan et al. (2015) carried out a wide-ranging exploration of the effects of environment, including host group mass, on galaxy properties, finding that the characteristic stellar mass increases with group mass. Barsanti et al. (2018) and Wang et al. (2018) have recently investigated the impact of GAMA group environment on star formation. Barsanti et al. (2018) find that the fraction of star-forming galaxies is higher in group outskirts where galaxies have recently been accreted, and lower in the central, virialized regions. Wang et al. (2018) find that, overall, star formation rate is suppressed in group environments relative to the field.

In this paper, we present galaxy LFs and SMFs as a function of host group mass, subdivided by galaxy colour, morphology, and by redshift. In Section 2 we describe the GAMA Galaxy Group Catalogue $\left(\mathrm{G}^{3} \mathrm{C}\right)$ and associated galaxy samples, as well as comparison mock catalogues and simulations. Section 3 describes the methods used to estimate the LFs and SMFs in bins of halo mass and redshift. Section 4 shows our results and we conclude in Section 5. In Appendix A we compare field LFs and SMFs between GAMA and mock and simulated samples. Appendix B investigates the effects of group-finding and halo mass estimation by comparing LFs using true mock groups and masses with those based on estimated quantities. We test our estimators on simulated data in Appendix C, showing that the $1 / V_{\max }$-weighted LF provides unbiased estimates, whereas the per-group CLF is biased in low-mass groups unless stringent redshift cuts are imposed.

For this work, we assume cosmological parameters of $\Omega_{M}=$ 
$0.3, \Omega_{\Lambda}=0.7$ with a Hubble constant of $H_{0}=100 h \mathrm{~km} \mathrm{~s}^{-1}$ $\mathrm{Mpc}^{-1}$. Group (halo) masses have been calibrated by weak lensing measurements, and are represented on a logarithmic scale by $\lg \mathcal{M}_{h} \equiv \log _{10}\left(\mathcal{M}_{h} / \mathcal{M}_{\odot} h^{-1}\right)$. Stellar masses in simulations, whose natural units are $\mathcal{M}_{\odot} h^{-1}$, are scaled by the relevant value of $h$ to be consistent with stellar masses for observed galaxies, so that both are represented by $\lg \mathcal{M}_{*} \equiv \log _{10}\left(\mathcal{M}_{*} / \mathcal{M}_{\odot} h^{-2}\right)$.

\section{GAMA DATA, MOCKS AND SIMULATIONS}

The GAMA project is a multi-wavelength spectroscopic galaxy survey based on an input catalogue described by Baldry et al. (2010). In this paper, we make use of the GAMA-II (Liske et al. 2015) equatorial fields, each of $12 \times 5$ degrees centred at $09 \mathrm{~h}$, $12 \mathrm{~h}$ and $14 \mathrm{~h} 30 \mathrm{~m} \mathrm{RA}$, called G09, G12 and G15 respectively. The GAMA-II Petrosian magnitude limit is $r<19.8$ mag for all three fields. This survey is complete in all regions with a completeness greater than $96 \%$ for all galaxies with up to 5 neighbours within 40 arcsec (see Liske et al. 2015, for a detailed description). We first discuss the GAMA mock catalogues, as these are used to justify our choice of group mass estimator.

\subsection{Mock catalogues and group mass estimates}

The GAMA mock catalogues have been designed to match GAMA-I survey data as closely as possible (updates to reflect the extended area of GAMA-II are currently in progress). These were constructed from the Millennium dark matter simulation (Springel et al. 2005) and populated with galaxies using the GALFORM (Bower et al. 2006) semi-analytic galaxy formation recipe. They are the same mocks used to tune and test the GAMA group-finding algorithm in Robotham et al. (2011, hereafter R11); readers are referred to that publication for further details of the mock GAMA group catalogues.

We compare the LFs of the GAMA mocks with GAMA data in Appendix A, finding that the characteristic magnitude of the mock galaxies is about 0.5 mag fainter than for GAMA galaxies. When comparing GAMA and mock grouped LFs, one should therefore focus on the trends with halo mass for each, rather than compare LF parameters.

Two mock group catalogues are available. The first, G3CMockHaloGroupv06, hereafter referred to as halo mocks, contains the positions and masses $\mathcal{M}_{\text {halo }}$ of the intrinsic haloes in the dark matter simulations. The second, G3CMOckFOFGroupv06, referred to as FoF mocks, has groups identified using the same friends-of-friends (FoF) algorithm, and masses estimated in the same way as for the GAMA data.

We compare two methods for estimating group masses. The first derives a dynamical mass $\mathcal{M}_{\text {dyn }}$ via the virial theorem from galaxy dynamics within each group (column MassA in the relevant group catalogue). The second derives a luminosity-based mass $\mathcal{M}_{\text {lum }}$ from group $r$-band luminosity (column LumB) using the weak-lensing calibrated scaling relation of Viola et al. (2015, eqn. 37). LumB provides the total $r$-band luminosity down to $M_{r}-5 \log _{10} h=-14$ mag in solar luminosities, multiplied by a constant calibration factor of $B=1.04$ (see R11 section 4.4 for details $)^{1}$.

1 The GAMA and mock group catalogues include an alternative group luminosity estimate, LumBfunc, in which the calibration factor $B$ is a function of redshift and group multiplicity. However, the GAMA and

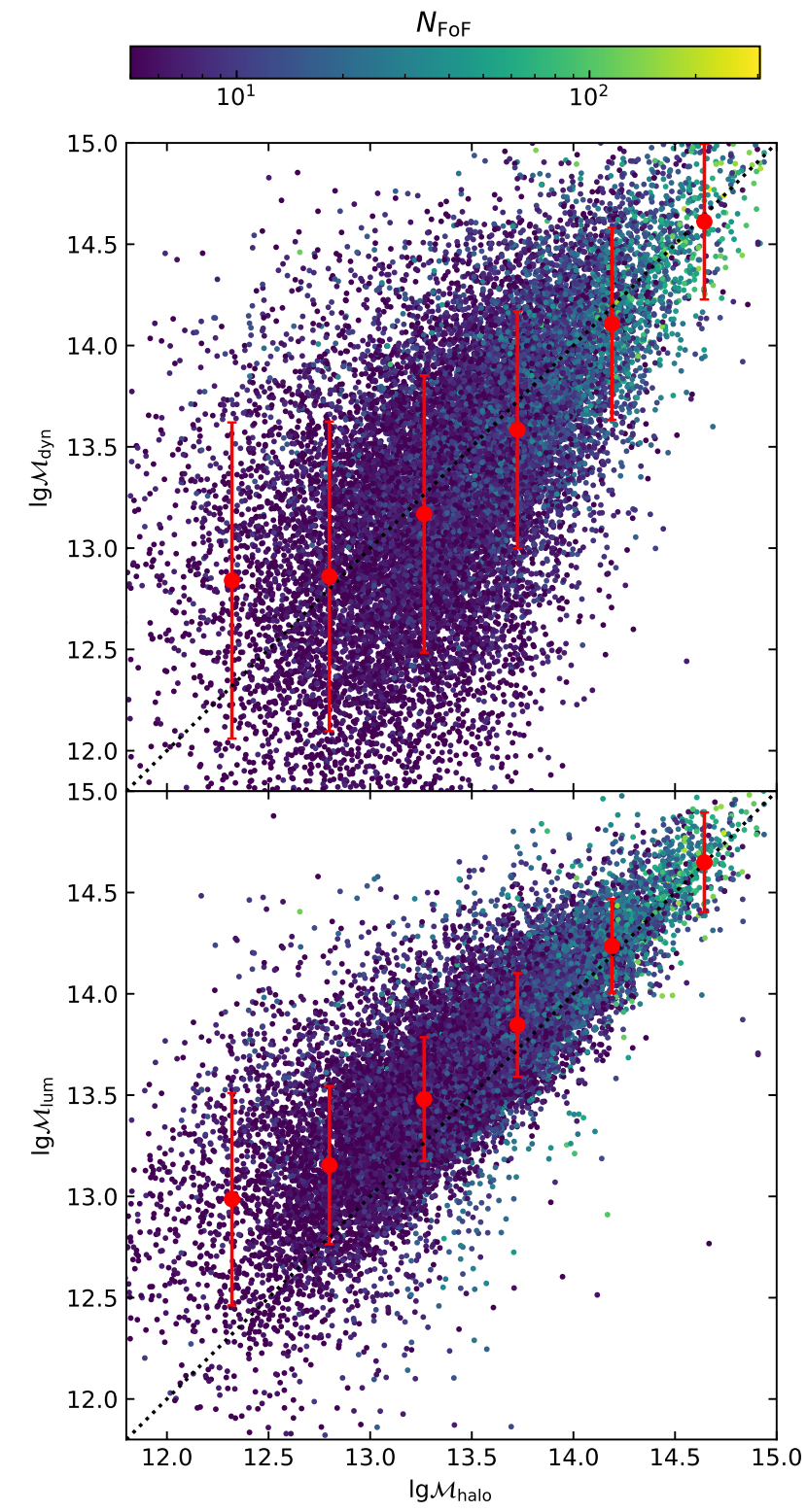

Figure 1. Comparison of luminosity-based ( $\lg \mathcal{M}_{\text {lum }}$, lower panel), and dynamical (lg $\mathcal{M}_{\text {dyn }}$, upper panel), estimates of mock group mass, against true mock halo mass, $\lg \mathcal{M}_{\text {halo }}$, colour coded by group membership. See text for details of these mass estimates. The red error-bars show mean and standard deviation of estimated halo mass in 0.5 mag bins of $\lg \mathcal{M}_{\text {halo }}$.

In order to check the reliability of these mass estimates, we match groups in the mock halo catalogue with those in the mock FoF catalogue on the basis of sharing the same iterative centre (see R11 section 4.2 for the definition of this). As for the real GAMA groups, we select only mock FoF groups with five or more members, as these richer groups are found to be the most reliable (R11). We also exclude groups for which less than $90 \%$ of the group

mock groups show significantly discrepant distributions of LumBfunc, with mock galaxies being on average about 1.6 times more luminous than GAMA galaxies. We also note (Margot Brouwer, private communication) that the Viola et al. (2015) scaling relations use LumB and not LumBfunc. 
is estimated to lie within the survey boundaries, i.e. we require GroupEdge $>0.9$. We can then compare the luminosity- and dynamically-based mass estimates from the FoF catalogue with the true halo masses from the halo catalogue. In Fig. 1, we see that the luminosity-based masses (lower panel) show a better correlation with halo mass than do the dynamical mass estimates (top panel), in agreement with the results of Han et al. (2015). We therefore use only the luminosity-based mass estimates in this paper. We note that both estimators are biased high at low halo masses, with a more pronounced bias for $\mathcal{M}_{\text {lum }}$ due to its smaller scatter. This suggests that the FoF group finder is tending to include spurious members in lower-mass groups, a perhaps not unexpected result given that the FoF linking length is independent of halo mass (cf. the halobased group finder used for the Yang et al. 2007 group catalogue, in which linking length scales with halo mass). When interpreting the dependence of galaxy luminosity on halo mass, one should also bear in mind that estimated halo mass is based on integrated galaxy luminosity. This circular logic is also true of previous work (e.g. Yang et al. 2008, 2009).

Uncertainties on mock LF estimates are determined from the scatter between nine independent realisations of the GAMA-I survey volume (each realisation comprising three $12 \times 4$ deg regions; 20 per cent smaller than the GAMA-II equatorial fields). Mock galaxies are taken from G3CMockGalv06. Absolute magnitudes are $K$-corrected (to redshift zero) with universal $K$ - and $e$-corrections as specified in Sec. 2.2 of R11. These GAMA mocks do not provide colour or morphological information for the galaxies, and so we present only 'total' mock LFs, without subdivision by colour or Sérsic index. Neither do these mock catalogues include stellar mass estimates, so we are unable to compare SMFs. Instead, we compare SMFs with the L-GALAXIES semi-analytic model, and two hydrodynamical simulations, described below.

\subsection{GAMA group data}

The GAMA Galaxy Group Catalogue $\left(\mathrm{G}^{3} \mathrm{Cv} 9\right)$ was generated using the GAMA-II spectroscopic survey and applying a friends-offriends (FoF) grouping algorithm; the first version of this catalogue $\left(\mathrm{G}^{3} \mathrm{Cv} 1\right)$ is presented by R11 using the GAMA-I survey. The $\mathrm{G}^{3} \mathrm{Cv} 9$ (hereafter abbreviated to $\mathrm{G}^{3} \mathrm{C}$ ) catalogue contains a total of 23,654 groups (comprising 2 or more members) containing a total of 75,029 galaxies; 40\% of GAMA galaxies are assigned to groups. As for the mocks, we utilise only groups which have five or more member galaxies and GroupEdge $>0.9$. This leaves us with a sample of 24,832 galaxies in 2,718 groups.

Masses are estimated from group luminosities LumB via the Viola et al. (2015) scaling relation, as discussed in the previous subsection. The mass-redshift distribution of our selected GAMA groups is shown in Fig. 2. There is a clear selection bias against finding low-mass groups at high redshift, demonstrating a strong correlation between group mass and the $r$-band luminosity of its fifth-brightest member. It is also, unsurprisingly, apparent that higher-mass groups tend to have more observed galaxy members. Groups at higher redshift for fixed mass tend to have fewer members, simply due to the $r<19.8$ mag flux limit of the GAMA-II survey.

We sub-divide the groups into four mass bins as defined in Table 1, chosen to provide roughly comparable numbers of galaxies. Comparing the halo and FoF mock groups, it is clear that the FoF algorithm is systematically overestimating the numbers of groups in all mass bins. It seems likely that the higher numbers of FoF cf. halo groups is due to the FoF algorithm aggregating lower-

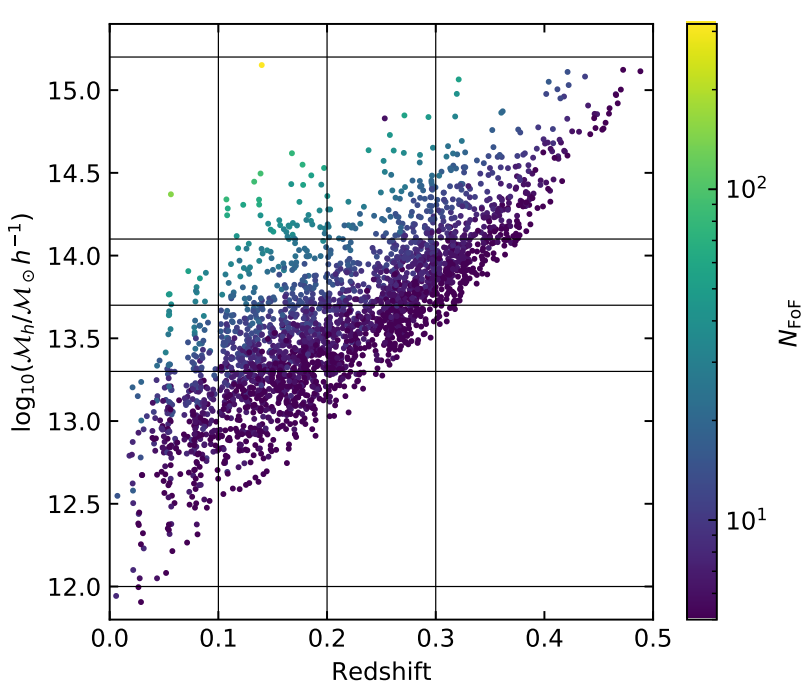

Figure 2. Mass-redshift distribution for GAMA groups that satisfy our selection criteria. Colour-coding indicates the number of group members on a logarithmic scale. The horizontal lines delineate the halo mass bins used in this analysis and the vertical lines show the redshift bins used when investigating LF evolution.

mass haloes, which individually would not satisfy our selection criteria, into one system. Altogether, the FoF mock catalogue contains about 20 per cent more groups with five or more members than does the halo mock catalogue. The numbers of GAMA group in each bin lie somewhere between the halo and FoF mocks, bearing in mind the 20 per cent smaller volume of the mocks.

In Appendix B, we investigate the effects of FoF group finding and luminosity-based mass estimation, by comparing LFs obtained from halo and FoF mock catalogues. We find that while the halo and FoF non-parametric LFs show qualitatively similar behaviour, they are formally inconsistent in all but the lowest mass bin, and with Schechter parameters that disagree by about $1-3 \sigma$. It is likely that our GAMA results will suffer from similar biases.

\subsection{Galaxy data}

\subsubsection{Central versus satellite}

Galaxies assigned to each group are ranked according to distance from the iterative centre of the group (R11, section 4.2.1). We define the first-ranked galaxy in each group as the central galaxy ( 95 per cent of the time this corresponds to the brightest galaxy), and all other galaxies as satellites, so that each group has one central galaxy and four or more satellites. Note that the GAMA group catalogue is constructed using a friend-of-friend (FoF) algorithm, whereas the SDSS group catalogue of Yang et al. (2007) is constructed using a halo-based method. As discussed by Robotham et al. (2010), the latter algorithm results in groups typically containing smaller numbers of galaxies, including groups that comprise a single galaxy, and so our results for central and satellite galaxies are not directly comparable with those of Yang et al. $(2008,2009)$. One could choose to treat ungrouped galaxies in the $\mathrm{G}^{3} \mathrm{C}$ as isolated centrals, but their host halo properties would be extremely uncertain. 
Table 1. Group bin names and log-mass limits, number of groups and galaxies, mean log-mass, and mean redshift for GAMA-II groups, intrinsic mock haloes, and FoF mock groups. Note that each mock realisation has about 20 per cent smaller volume than the GAMA-II equatorial fields.

\begin{tabular}{|c|c|c|c|c|c|c|c|c|c|c|c|c|c|}
\hline & \multirow[b]{2}{*}{$\lg \mathcal{M}_{h, \text { limits }}$} & \multicolumn{4}{|c|}{ GAMA } & \multicolumn{4}{|c|}{ Halo Mocks } & \multicolumn{4}{|c|}{ FoF Mocks } \\
\hline & & $N_{\text {grp }}$ & $N_{\mathrm{gal}}$ & $\overline{\lg \mathcal{M}_{h}}$ & $\bar{z}$ & $N_{\text {grp }}$ & $N_{\mathrm{gal}}$ & $\overline{\lg \mathcal{M}_{h}}$ & $\bar{z}$ & $N_{\text {grp }}$ & $N_{\mathrm{gal}}$ & $\overline{\lg \mathcal{M}_{h}}$ & $\bar{z}$ \\
\hline $\mathcal{M 1}$ & {$[[12.0,13.3]$} & 712 & 4520 & 13.03 & 0.12 & 441 & 3133 & 12.98 & 0.12 & 584 & 3914 & 12.97 & 0.12 \\
\hline $\mathcal{M} 2$ & {$[13.3,13.7]$} & 856 & 6817 & 13.50 & 0.19 & 594 & 4971 & 13.51 & 0.19 & 744 & 5676 & 13.51 & 0.19 \\
\hline $\mathcal{M} 3$ & {$[13.7,14.1]$} & 722 & 6944 & 13.88 & 0.26 & 567 & 6146 & 13.88 & 0.25 & 668 & 6705 & 13.89 & 0.26 \\
\hline M4 & {$[14.1,15.2]$} & 422 & 6762 & 14.37 & 0.32 & 310 & 7688 & 14.34 & 0.29 & 353 & 6868 & 14.34 & 0.30 \\
\hline
\end{tabular}

\subsection{2 r-band luminosities}

Our $r$-band LFs are derived from SDSS DR7 Petrosian magnitudes, corrected for Galactic extinction using the dust maps of Schlegel et al. (1998). Since galaxies are observed at different redshifts, a correction to the intrinsic luminosity has to be applied according to the rest frame of the galaxy. All galaxies in this analysis have been corrected by the so-called $K$-correction (Humason et al. 1956) using the KCORRECT V4_2 code (Blanton et al. 2003; Blanton \& Roweis 2007) employing the SExtractor (Bertin \& Arnouts 1996) AUTO magnitudes reported in ApMatchedCatv06 (Driver et al. 2016). These $K$-corrections were obtained from the GAMA data management unit (DMU) kCorrectionsv05 (Loveday et al. 2015). In order to be compatible with results from the GAMA mocks and hydrodynamical simulations, we $K$-correct to redshift zero $^{2}$. Absolute magnitudes in this band are indicated by ${ }^{0.0} M_{r}$.

When not subdividing into redshift bins, we apply a luminosity evolution correction of $+Q_{e} z$ mag, where $Q_{e}=1.0$. In principle, one might expect evolution to be environment-dependent, but due to degeneracies when simultaneously fitting for luminosity evolution, density evolution, and large-scale structure density variations (see Loveday et al. 2015), we assume global evolution corrections. See Section 3.1 for more details on these evolution corrections.

\subsubsection{Stellar masses}

Galaxy stellar masses are obtained from the GAMA DMU Stel larMasses Lambdarv20 (Taylor et al. 2011). The stellar masses given in this table are based on LAMBDAR matched aperture photometry (Wright et al. 2016). We apply a correction for aperture to total flux using the fluxscale parameter, which gives the ratio of total (Sérsic) to LAMBDAR flux. We use those 96 per cent of galaxies with a physically reasonable value of the fluxscale parameter, that is in the range 0.8-10. See Wright et al. (2017) for a comparison of these stellar mass estimates, based on optical to near-IR photometry, with alternative estimates made using MAGPHYS (da Cunha et al. 2008; da Cunha \& Charlot 2011), as well as a comprehensive discussion of possible systematic errors affecting stellar mass estimates.

\subsubsection{Colour}

$\mathrm{G}^{3} \mathrm{C}$ member galaxies are separated into red and blue populations using restframe and dust-corrected $(g-i)^{*}$ intrinsic stellar colours from the StellarMassesLambdarv20 DMU (Taylor et al. 2011). In Fig. 3 we plot $(g-i)^{*}$ colour versus log stellar mass in four redshift slices. The red line is a linear dividing line, fit by eye,

2 Although simulation snapshots are at higher redshifts, the photometric bands are rest-frame.

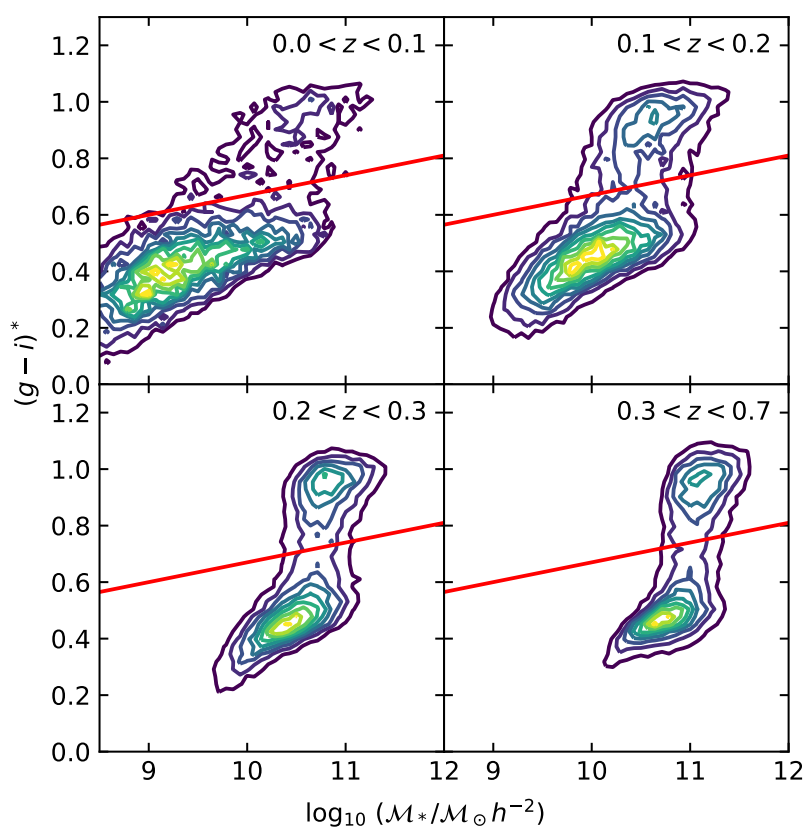

Figure 3. $(g-i)$ intrinsic stellar colour versus log stellar mass in four redshift slices as labelled. Contours are linearly spaced in density. The red line shows our blue/red division given by equation (1).

which roughly follows the division between ' $R$ ' and ' $B$ ' galaxies in Fig. 11 of Taylor et al. (2015), and is given by

$(g-i)^{*}=0.07 \log _{10}\left(\mathcal{M}_{*} / \mathcal{M}_{\odot} h^{-2}\right)-0.03$.

Fig. 3 demonstrates that this cut is applicable over the full redshift range of the GAMA-II survey, and has the advantage that it is corrected for internal dust-reddening. We note that with this definition, there are very few red galaxies at low redshift, $z<0.1$. Taylor et al. (2015) argue that a probablistic assignment of galaxies to ' $R$ ' and ' $\mathrm{B}$ ' populations is preferable to a hard (and somewhat arbitrary) red/blue cut. However, for our purposes, dividing the galaxy population into star-forming and quiescent using a hard cut, is quite adequate, and certainly a lot simpler than applying the Taylor et al. (2015) 40-parameter probabilistic model (which has been tuned for nearby $z<0.12$ galaxies).

\subsubsection{Morphology}

The morphology of galaxies is fundamental to understanding their behaviour at different evolutionary epochs. We are therefore interested in comparing spheroidal and disky galaxy shapes with colour. Generally, red colour is associated with galaxies containing a low fraction of dust and low star formation, i.e. early type or spheroid- 


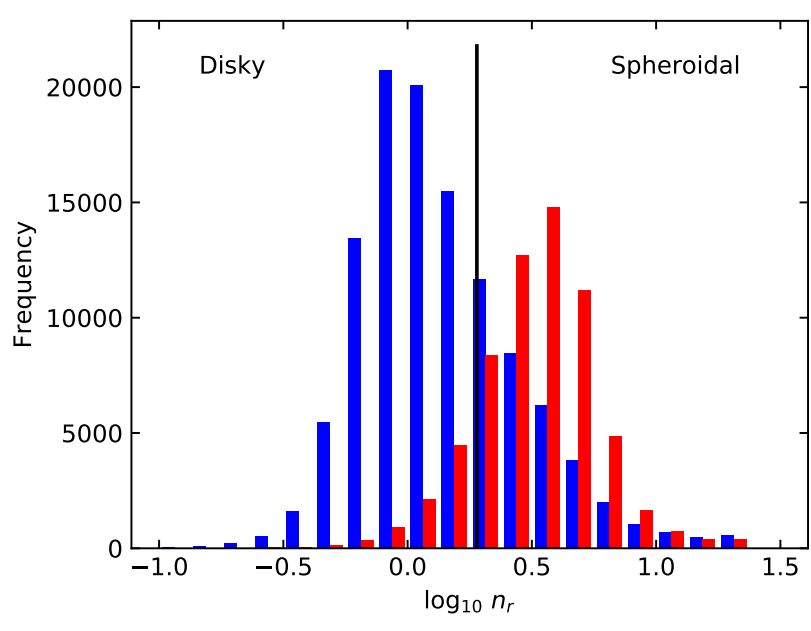

Figure 4. Histogram of GAMA-II $\log r$-band Sérsic index $n_{r}$ colour-coded by classification into blue and red galaxies. The vertical black line shows the separation into disky and spheroidal at $n_{r}=1.9$. While the majority of blue and red galaxies lie to the left and right of this line, respectively, there are significant numbers of blue galaxies with high index, and vice-versa.

als, while the blue population is usually associated with star forming galaxies or late types, mainly spirals.

The LF (Kelvin et al. 2014a) and SMF (Kelvin et al. 2014b; Moffett et al. 2016) have been presented for galaxies separated into five bins of morphological type using the GAMA VisualMorphology DMU. However, these visual morphologies are only available for a very local sample $(z<0.06)$. Many techniques have been developed to make an objective classification and also to classify thousands of galaxies automatically (e.g. Huertas-Company et al. 2015); however, these methods work well only with highly-resolved images. At the moment, GAMA does not have images with sufficient resolution at $z \gtrsim 0.15$. Simple methods, using the Sérsic index (Sérsic 1963), give a reliable classification at least to distinguish between spheroidal and disk-dominated galaxies (e.g. Barden et al. 2005). Therefore, we have made a simple classification based on the $r$-band Sérsic index, $n_{r}$, taken from the GAMA DMU SersicCat SD S.Sv0 9 (Kelvin et al. 2012). Galaxies are considered as spheroidal (or high- $n$ ) when $n_{r}>1.9$ and disky (or low- $n$ ) when $n_{r}<1.9$. Many authors take the cut to be 2.5 (e.g. Barden et al. 2005); however, Kelvin et al. (2012) show in their Fig. 15 that the GAMA Sérsic index distribution in the $r$-band is bi-modal, with a minimum at $n_{r}=1.9$. We show a histogram of $\log r$-band Sérsic index colour-coded by classification into blue and red galaxies in Fig. 4. While the majority of blue and red galaxies correspond to disky and spheroidal respectively, there are significant numbers of blue galaxies with high index, and vice-versa.

While the GAMA Sérsic modelling takes account of the image point spread function, one might still worry that galaxies observed at higher redshift are less likely to be resolved in SDSS imaging, and thus might have their Sérsic indices biased low (a Gaussian profile corresponds to $n=0.5$ ). To test for this, in Fig. 5 we plot the fractions of luminous $\left(-22.5 \leq M_{r}<-21.5\right)^{3}$ field galaxies classified as either spheroidal or as red by our above cuts, in $\Delta z=0.05$

3 Without applying these luminosity limits, the red and spheroidal fraction both strongly increase with redshift, since high-redshift galaxies tend to be more luminous in a flux-limited sample. We choose to show luminous

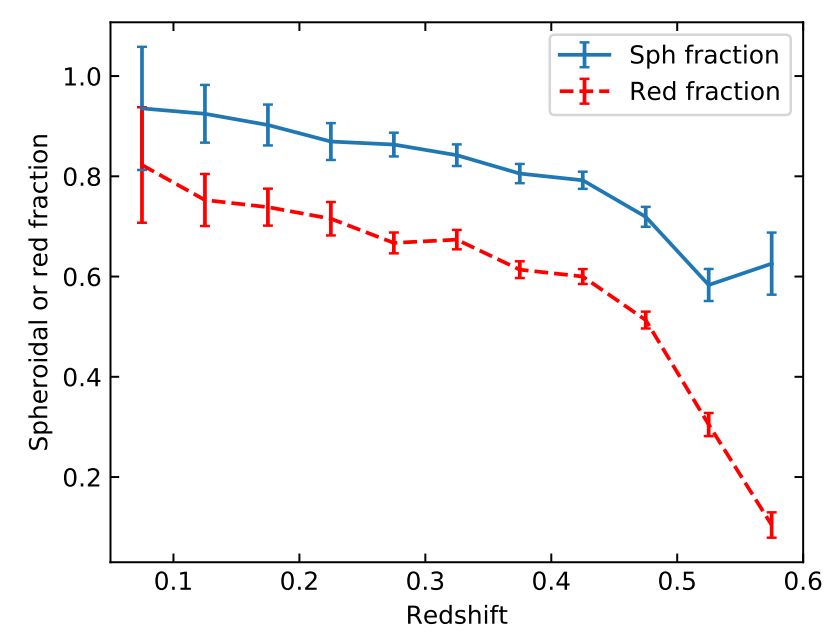

Figure 5. Fraction of luminous $\left(-22.5 \leq M_{r}<-21.5\right)$ field galaxies classified as spheroidal (blue solid line) or red (red dashed line) in $\Delta z=$ 0.05 bins of redshift. For $z<0.5$, one sees that the spheroidal fraction closely tracks the red fraction, thus suggesting any bias in measured Sérsic index with redshift is minimal.

bins of redshift. For $z<0.5$, corresponding to the redshift limit of our group sample, one sees that the spheroidal fraction closely tracks the red fraction, thus suggesting any bias in Sérsic index with redshift is minimal.

\subsubsection{Completeness}

Loveday et al. (2012) discuss three sources of incompleteness in GAMA-I data: incompleteness in the SDSS input catalogue (primarily a function of surface brightness), incompleteness in GAMA target selection, and redshift failures. For the $r$-band LF, target completeness is essentially 100\% (Loveday et al. 2012). Therefore, we correct only for input catalogue incompleteness and redshift failures, following the GAMA-II updates of Loveday et al. (2015).

GAMA sample selection is complete in $r$-band magnitude, but not in stellar mass - blue galaxies are visible to higher redshifts than red galaxies. We determine stellar mass completeness as a function of redshift following a simplified version of the method described in Appendix C of Wright et al. (2017). One would expect the SMF to keep rising to lower masses (at least down to $\lg M_{*} \sim 8$ or so), and so we estimate mass completeness by locating the turnover point in stellar mass density as a function of redshift.

Fig. 6 shows a scatter-plot of log galaxy stellar mass against redshift for our sample of grouped GAMA galaxies. Galaxies are colour-coded according to intrinsic $(g-i)^{*}$ colour as indicated. We consider ten equally-spaced bins in redshift, ranging from $z=0.0$ to $z=0.5$. Within each redshift bin, we determine the kernel density estimate (KDE) of $\lg \mathcal{M}_{*}$, using a Gaussian smoothing kernel and default bandwidth as determined by the routine scipy.stats.gaussian_kde. The turn-over point in stellar mass, $\lg \mathcal{M}_{*}^{t}$, is then chosen as the maximum of the KDE. Uncertainty in $\lg \mathcal{M}_{*}^{t}$ is estimated by recalculating the $\mathrm{KDE}$ for 100 bootstrap samples of the $\lg \mathcal{M}_{*}$ data in each redshift bin. These

galaxies since this is where we see domination by spheroidal systems in the group LFs. 


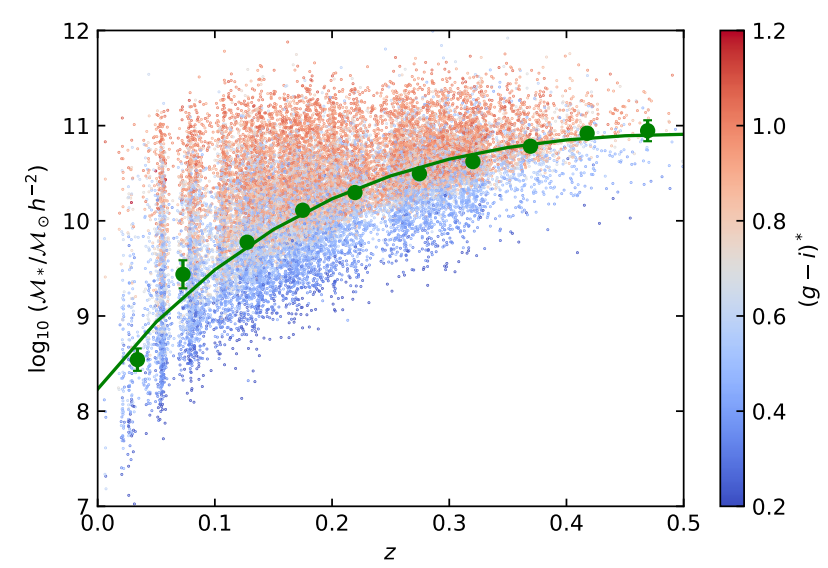

Figure 6. Scatter-plot of galaxy stellar mass against redshift for grouped GAMA galaxies. Galaxies are colour-coded according to intrinsic $(g-i)^{*}$ colour as indicated. Large symbols indicate the turnover point in log stellar mass density $\lg \mathcal{M}_{*}^{t}$ and its standard deviation in bins of redshift. The line shows a second-order polynomial best-fit relation between $\lg \mathcal{M}_{*}^{t}$ and scale factor $a=1 /(1+z)$.

turn-over points and uncertainties are indicated by the large symbols with error bars. Finally, we fit a second-order polynomial to $\lg \mathcal{M}_{*}^{t}$ as a function of scale factor ${ }^{4} a=1 /(1+z)$. We do not inverse-variance weight the $\lg \mathcal{M}_{*}^{t}$ estimates in this fit, as the very small uncertainties in $\lg \mathcal{M}_{*}^{t}$ at intermediate redshifts result in overfitting to intermediate bins and poor fit behaviour at low and high redshifts. The polynomial fit, shown by the curve, is given by

$\lg \mathcal{M}_{*}^{t}=1.17+29.69 a-22.58 a^{2}$.

SMF estimates include only galaxies above this mass limit; equation (2) is also used to determine the visibility of a galaxy of given stellar mass in the SMF estimate (see section 3).

\subsection{SMF comparison simulations}

We compare our GAMA grouped SMF results with predictions from the L-GALAXIES semi-analytic model (SAM, Henriques et al. 2015) and from two recent hydrodynamical simulations EAGLE and Illustris TNG. For all three models/simulations, we utilise data cubes at single snapshot redshifts corresponding roughly to the mean redshift of the GAMA data, $\bar{z} \approx 0.2$, rather than attempting to create mock light-cones. This results in a much higher abundance of low-mass haloes than observed in GAMA data, and so we set halo mass bin limits to give approximately the same mean halo mass as for the GAMA data, see Table 2.

For the L-GALAXIES SAM, which is based on the Millennium (Springel et al. 2005) and Millennium-II (Boylan-Kolchin et al. 2009) N-body simulations, we select the closest redshift snapshot to the mean GAMA redshift individually for each halo bin. Halo mass is defined by the mass within an overdensity of 200 times the critical density.

From the EAGLE suite of simulations (Crain et al. 2015; Schaye et al. 2015) we utilise snapshot $26(z=0.18)$ from the largest-volume simulation, Ref-L0100N1504. We use

4 We found that a quadratic function provides a better fit to scale factor than to redshift.
Group_M_Mean200 from the FOF table for halo mass, and Mass_Star from the $30 \mathrm{kpc}$ Aperture table, for stellar mass; see McAlpine et al. (2016) for a complete description of the EAGLE database.

From the suite of IllustrisTNG hydrodynamical simulations (Marinacci et al. 2018; Naiman et al. 2018; Nelson et al. 2018, 2019; Pillepich et al. 2018; Springel et al. 2018), we use the full-resolution simulation with the largest box-size of $300 \mathrm{Mpc}$ (205 $h^{-1} \mathrm{Mpc}$ for $\left.h=0.6774\right)$, TNG300-1, at redshift $z=0.2$ (snapshot 84). Halo masses are given by the FoF halo parameter Group_M_Mean200, and stellar masses are obtained from the subhalo parameter SubhaloMass InRadType for particle type 4 (stars), the stellar masss within twice the stellar half mass radius. As recommended by Pillepich et al. (2018, A1), we multiply the given stellar masses by a resolution correction factor of 1.4, appropriate for haloes in the mass range $12<\lg \mathcal{M}_{h}<15$.

We subdivide the IllustrisTNG galaxies into blue and red using the colour cut

$(g-i)^{*}=0.07 \log _{10}\left(\mathcal{M}_{*} / \mathcal{M}_{\odot} h^{-2}\right)+0.24$,

where $(g-i)^{*}$ is the intrinsic stellar colour determined from the subhalo parameters Subhalostellarphotometrics, and $\mathcal{M}_{*}$ is the resolution-corrected stellar mass. This is the same as equation (1) used to select blue and red GAMA galaxies, except that we have adjusted the zero-point offset, so that equation (3) better follows the 'green valley' in IllustrisTNG galaxy colours.

In order to assess the consistency of these simulations with GAMA data, we compare field (i.e. group-independent) LFs and SMFs in Appendix A. We find that the IllustrisTNG LF underpredicts the numbers of low- and high-luminosity galaxies. SMFs are in better agreement, although IllustrisTNG over-predicts the numbers of very massive $\left(\lg \mathcal{M}_{*} \gtrsim 11\right)$ galaxies.

\section{METHODS}

In this section we describe our methods for estimating the LF and SMF from GAMA data and mock catalogues; these estimates are trivial for the simulations, since they come in the form of volumelimited boxes. For GAMA data, uncertainties are determined from nine jackknife samples, each comprising $4 \times 5$ deg of contiguous area. These yield larger uncertainties than given by assuming Poisson errors. For mock catalogues, uncertainties come from the scatter between nine independent realisations.

\subsection{LF and SMF estimators}

We first determine the limiting redshift $z_{\lim }$ of each galaxy in the sample. For the LF calculation, $z_{\lim } \equiv z_{\lim }^{\operatorname{lum}}$ is determined by the GAMA survey magnitude limit of $r=19.8 \mathrm{mag}$, the galaxy's absolute r-band magnitude, and its redshift-dependent $K-$ and $e$-corrections. For the SMF calculation, $z_{\lim }=\min \left(z_{\lim }^{\operatorname{lum}}, z_{\lim }^{\operatorname{mass}}\right)$, where $z_{\lim }^{\text {mass }}$ is obtained by substituting the galaxy's mass for $\mathcal{M}_{*}^{t}$ in equation (2) and solving for redshift.

We estimate the LFs and SMFs using a density-corrected $V_{\max }$ estimator, allowing for the fact that GAMA groups have a minimum membership threshold of $N_{t}$ galaxies, where, for this analysis, we have chosen $N_{t}=5$. The limiting redshift $z_{\lim , j}$ of group $j$ corresponds to $z_{\text {lim }}^{\text {lum }}$ of its $N_{t}$ th brightest member: beyond this redshift the group would drop below the membership threshold, and hence be excluded from the sample. Thus the correct limiting redshift to apply to each galaxy $i$ in group $j$ is $z_{\max , i}=\min \left(z_{\lim , i}, z_{\lim , j}\right)$. Here 
Table 2. Halo samples for L-GaLAXIES, EAGLE and IllustrisTNG simulations. The log-mass limits (second column) are chosen to give mean log masses close to those of GAMA galaxies in corresponding halo mass bins (see Table 1). For each simulation, we give the number of haloes and galaxies, mean $\log$-mass, and snapshot redshift. The number of galaxies quoted for L-GALAXIES comprises only those from Millennium, not Millennium II, i.e. those with $\lg \mathcal{M}_{*}>$ 9.5. EAGLE and IllustrisTNG samples give the number of galaxies with $\lg \mathcal{M}_{*}>8.5$.

\begin{tabular}{|c|c|c|c|c|c|c|c|c|c|c|c|c|c|}
\hline & \multirow[b]{2}{*}{$\lg \mathcal{M}_{h, \text { limits }}$} & \multicolumn{4}{|c|}{ L-GALAXIES } & \multicolumn{4}{|c|}{ EAGLE } & \multicolumn{4}{|c|}{ IllustrisTNG } \\
\hline & & $N_{\text {halo }}$ & $N_{\mathrm{gal}}$ & $\overline{\lg \mathcal{M}_{h}}$ & $z$ & $N_{\text {halo }}$ & $N_{\text {gal }}$ & $\overline{\lg \mathcal{M}_{h}}$ & $z$ & $N_{\text {halo }}$ & $N_{\text {gal }}$ & $\overline{\lg \mathcal{M}_{h}}$ & $z$ \\
\hline $\mathcal{M} 1$ & {$[12.8,13.3]$} & 44665 & 201538 & 13.00 & 0.11 & 155 & 1612 & 13.02 & 0.18 & 3713 & 30570 & 13.05 & 0.20 \\
\hline $\mathcal{M} 2$ & {$[13.3,13.7]$} & 11906 & 134384 & 13.47 & 0.18 & 42 & 1069 & 13.47 & 0.18 & 1040 & 21909 & 13.50 & 0.20 \\
\hline $\mathcal{M} 3$ & {$[13.7,14.1]$} & 3665 & 93949 & 13.86 & 0.26 & 9 & 644 & 13.85 & 0.18 & 405 & 19055 & 13.89 & 0.20 \\
\hline $\mathcal{M} 4$ & {$[14.1,14.8]$} & 910 & 60985 & 14.29 & 0.31 & 5 & 950 & 14.31 & 0.18 & 120 & 15468 & 14.37 & 0.20 \\
\hline
\end{tabular}

$z_{\lim , i}$ is the limiting redshift of galaxy $i$ determined as described in the first paragraph of this sub-section, i.e. neglecting the requirement that its host group be selected.

For a sample bounded by redshift limits $\left(z_{\mathrm{lo}}, z_{\mathrm{hi}}\right)$, we weight galaxy $i$ by $1 / V_{\max , i}^{\mathrm{dc}}$, where

$V_{\max , i}^{\mathrm{dc}}=\int_{z_{\mathrm{lo}}}^{\min \left(z_{\mathrm{hi}}, z_{\max , i}\right)} \Delta(z) P(z) V(z) d z$.

In this equation, $\Delta(z)$ is the relative overdensity (taken from fits to the entire GAMA-II sample ${ }^{5}$ by Loveday et al. 2015), $P(z)=$ $P(0) 10^{0.4 P_{e} z}$ parametrizes number density evolution, and $V(z)$ is the comoving volume element at redshift $z$. This estimator has been derived by maximum likelihood (Cole 2011; Loveday et al. 2015) and provides a straightforward way of accounting for both density fluctuations and redshift evolution within the galaxy sample being analysed.

Higher-mass groups tend to be found at higher redshift (Fig. 2), and so to separate the effects of redshift evolution and environment, we apply evolution corrections parametrized by $Q_{e}=$ $1, P_{e}=1$ for luminosity and density evolution respectively. The corrected absolute magnitude is given by $M_{c}=M+Q_{e} z$ and the density evolution parameter $P_{e}$ is defined in the preceding paragraph (see also Lin et al. 1999; Loveday et al. 2015). To first order, these corrections will take out evolutionary effects so as to isolate the effects of environment on the LF.

To estimate the LF and SMF for a given sample of galaxies, we simply count galaxies in bins of absolute $r$-band magnitude or $\lg \mathcal{M}_{*}$, respectively, weighting each galaxy by its $1 / V_{\max }^{\mathrm{dc}}$.

Our LF estimator is tested in Appendix C, and compared with estimates of the CLF (number of galaxies per group, rather than per unit volume). We find that that unbiased LFs may be estimated without applying redshift cuts, whereas the CLF estimator will overestimate the number of luminous galaxies unless a volumelimited group sample is defined, which would severely reduce the sample size. For this reason, we show only LF and SMF results, and not their conditional (per-group) variants, the CLF and CSMF.

\subsection{Functional fits}

Following Yang et al. (2008, 2009), we fit log-normal functions to the LFs and SMFs of central galaxies, and Schechter functions to those of satellite galaxies.

Explicitly, the log-normal LFs and SMFs take the form

$\phi_{c}(M)=\phi_{c}^{*} \exp \left[-\frac{\left(M-M_{c}\right)^{2}}{2 \sigma_{c}^{2}}\right]$,

5 In principle, one should use $\Delta(z)$ for each sub-sample considered, but since these $\Delta(z)$ estimates would be noisy, we make the first-order assumption that radial overdensities of different samples vary in the same way. where $\phi_{c}^{*}, M_{c}$ and $\sigma_{c}$ correspond to the peak height, central value and standard deviation of the distribution respectively, and $M$ refers either to magnitude (LF) or log mass (SMF).

Satellite galaxies may be fit by generalised Schechter functions of the form

$\phi_{S}(L) d L=\phi_{s}^{*}\left(\frac{L}{L^{*}}\right)^{\alpha} \exp \left[-\left(\frac{L}{L^{*}}\right)^{\beta}\right] d\left(\frac{L}{L^{*}}\right)$,

where $L$ is either luminosity (LF) or stellar mass (SMF), $\phi_{S}^{*}$ is the normalisation, $L^{*}$ the characteristic luminosity or stellar mass, and $\alpha$ the faint-end or low-mass slope, such that $\alpha=-1$ corresponds to fixed number density per unit magnitude or per unit log-mass. The parameter $\beta$, the power to which $L / L^{*}$ is raised within the exponential, varies the rate at which the function drops at the bright/highmass end. Yang et al. $(2008,2009)$ use $\beta \equiv 2$ to fit their satellite LFs and SMFs. We instead use a standard Schechter function, with $\beta \equiv 1$, since that gives a slightly better fit (smaller $\chi^{2}$ values) to our results. While fits are improved further if we allow $\beta$ to vary as a free parameter, the strong degeneracy between $L^{*}$ and $\beta$ makes any trends with halo mass difficult to interpret.

We fit to LFs over the range of absolute magnitudes $-24<$ ${ }^{0.0} M_{r}<-16$, and to SMFs over the mass range $9.0<\lg \mathcal{M}_{*}<$ 12.5. While there are some reliable GAMA SMF measurements for $\lg \mathcal{M}_{*}<9.0$, the simulations, particularly IllustrisTNG, are not fully resolved below this mass limit.

When tabulating functional fits, we quote non-marginalized 1$\sigma$ errors on the parameters. For likelihood plots of the shape parameters, we show 1- $\sigma$ likelihood contours, but now marginalize over the normalisation parameter $\phi^{*}$.

\subsection{Redshift evolution}

In order to investigate evolution in the LF and SMF, we subdivide the sample into three redshift slices given by $z=[0.002,0.1]$, [0.1, $0.2]$ and $[0.2,0.3]$. From Fig. 2, we see that the group catalogue is approximately complete to redshift $z=0.3$ for groups of mass $\lg \mathcal{M}_{h} \approx 13.7$ and higher - see also Appendix C. We therefore use only mass bins $\mathcal{M} 3$ and $\mathcal{M} 4$ when subdividing by redshift. Since we are now explicitly isolating evolutionary effects by subdividing the galaxies into redshift slices, we 'switch off' evolution corrections, that is we set the evolution parameters to $P_{e}=Q_{e}=0$.

When subdividing by redshift, it is necessary to set completeness limits on the luminosity and mass range on the LF and SMF, respectively, as discussed in Section 3.3 of Loveday et al. (2012). For the LF, we set a faint absolute magnitude limit given by assuming a $K$-correction at the lower redshift limit corresponding to the 95-th percentile of the subsample under analysis, thus assuring that the faintest bin used is at least 95 per cent complete. For the SMF, the stellar mass limit as a function of redshift is determined from equation (2). 
Table 3. Log-normal fits (equation 5) to the central galaxy LF for different galaxy samples as indicated. The final column gives the $\chi^{2}$ value and degrees of freedom $v$ of each fit; these fits are mostly good.

\begin{tabular}{|c|c|c|c|c|c|}
\hline & $N_{\text {gal }}$ & $M_{c}$ & $\sigma_{c}$ & $\lg \phi_{c}^{*}$ & $\chi^{2} / v$ \\
\hline \multicolumn{6}{|c|}{ Mock all } \\
\hline $\mathcal{M} 1$ & 584 & $-20.33 \pm 0.06$ & $0.75 \pm 0.03$ & $-3.33 \pm 0.05$ & $11.7 / 6$ \\
\hline $\mathcal{M} 2$ & 744 & $-21.61 \pm 0.02$ & $0.45 \pm 0.01$ & $-3.96 \pm 0.03$ & $16.0 / 8$ \\
\hline $\mathcal{M} 3$ & 668 & $-21.80 \pm 0.03$ & $0.47 \pm 0.02$ & $-4.41 \pm 0.03$ & $9.2 / 6$ \\
\hline $\mathcal{M} 4$ & 352 & $-22.10 \pm 0.04$ & $0.47 \pm 0.03$ & $-4.93 \pm 0.04$ & $7.7 / 6$ \\
\hline \multicolumn{6}{|c|}{ GAMA all } \\
\hline $\mathcal{M} 1$ & 699 & $-20.71 \pm 0.08$ & $0.55 \pm 0.04$ & $-3.01 \pm 0.06$ & $3.9 / 5$ \\
\hline $\mathcal{M} 2$ & 842 & $-21.70 \pm 0.02$ & $0.43 \pm 0.01$ & $-3.95 \pm 0.03$ & $2.5 / 3$ \\
\hline $\mathcal{M} 3$ & 699 & $-22.03 \pm 0.02$ & $0.41 \pm 0.01$ & $-4.39 \pm 0.03$ & $8.6 / 3$ \\
\hline $\mathcal{M} 4$ & 392 & $-22.35 \pm 0.02$ & $0.47 \pm 0.02$ & $-4.97 \pm 0.03$ & $3.5 / 2$ \\
\hline \multicolumn{6}{|c|}{ GAMA blue } \\
\hline $\mathcal{M} 1$ & 215 & $-20.45 \pm 0.16$ & $0.64 \pm 0.09$ & $-3.39 \pm 0.11$ & $1.5 / 5$ \\
\hline $\mathcal{M} 2$ & 230 & $-21.51 \pm 0.03$ & $0.42 \pm 0.02$ & $-4.52 \pm 0.04$ & $2.6 / 3$ \\
\hline $\mathcal{M} 3$ & 189 & $-21.95 \pm 0.04$ & $0.46 \pm 0.03$ & $-5.03 \pm 0.06$ & $0.6 / 3$ \\
\hline $\mathcal{M} 4$ & 101 & $-22.30 \pm 0.06$ & $0.53 \pm 0.04$ & $-5.64 \pm 0.05$ & $0.6 / 2$ \\
\hline \multicolumn{6}{|c|}{ GAMA red } \\
\hline $\mathcal{M} 1$ & 484 & $-20.80 \pm 0.06$ & $0.51 \pm 0.03$ & $-3.24 \pm 0.06$ & $3.2 / 4$ \\
\hline $\mathcal{M} 2$ & 612 & $-21.77 \pm 0.02$ & $0.41 \pm 0.02$ & $-4.06 \pm 0.04$ & $3.1 / 3$ \\
\hline $\mathcal{M} 3$ & 510 & $-22.09 \pm 0.02$ & $0.41 \pm 0.02$ & $-4.53 \pm 0.03$ & $6.8 / 2$ \\
\hline $\mathcal{M} 4$ & 291 & $-22.37 \pm 0.02$ & $0.44 \pm 0.02$ & $-5.06 \pm 0.03$ & $5.7 / 2$ \\
\hline \multicolumn{6}{|c|}{ GAMA low-n } \\
\hline $\mathcal{M} 1$ & 106 & $-20.59 \pm 0.10$ & $0.41 \pm 0.05$ & $-3.51 \pm 0.17$ & $2.1 / 3$ \\
\hline $\mathcal{M} 2$ & 95 & $-21.44 \pm 0.06$ & $0.50 \pm 0.05$ & $-5.00 \pm 0.08$ & $0.2 / 3$ \\
\hline $\mathcal{M} 3$ & 67 & $-21.69 \pm 0.05$ & $0.41 \pm 0.03$ & $-5.42 \pm 0.03$ & $0.1 / 2$ \\
\hline $\mathcal{M} 4$ & 32 & $-21.92 \pm 0.12$ & $0.58 \pm 0.13$ & $-6.14 \pm 0.11$ & $0.3 / 2$ \\
\hline \multicolumn{6}{|c|}{ GAMA high-n } \\
\hline $\mathcal{M} 1$ & 593 & $-20.75 \pm 0.09$ & $0.55 \pm 0.04$ & $-3.12 \pm 0.06$ & $3.5 / 5$ \\
\hline $\mathcal{M} 2$ & 747 & $-21.74 \pm 0.02$ & $0.41 \pm 0.01$ & $-3.97 \pm 0.03$ & $0.7 / 3$ \\
\hline $\mathcal{M} 3$ & 632 & $-22.08 \pm 0.02$ & $0.39 \pm 0.01$ & $-4.41 \pm 0.03$ & $4.9 / 3$ \\
\hline $\mathcal{M} 4$ & 360 & $-22.38 \pm 0.02$ & $0.45 \pm 0.02$ & $-4.99 \pm 0.02$ & $2.2 / 2$ \\
\hline
\end{tabular}

\section{RESULTS}

\subsection{Group galaxy LF}

Our LF results, colour-coded by halo mass, are plotted in Fig. 7. Log-normal and Schechter parameter fits for central and satellite galaxies respectively are tabulated in Tables 3 and 4 .

\subsubsection{Central versus satellite}

Fig. 7 plots the LFs of central and satellite galaxies, in the left- and right-hand panel sets, respectively. Unsurprisingly, central galaxies dominate the bright-end of each LF, while satellites dominate the faint end. Due to the trend of increasing group membership with halo mass (Fig. 2), satellite galaxies become an increasingly dominant contributor to overall group luminosity as halo mass increases.

On the whole, central galaxy LFs are well fit by log-normal functions (Table 3), although the mock LFs are slightly skewed to lower luminosities. Schechter functions provide generally good fits to the satellite LFs, although sometimes they under-fit the faint-end in higher-mass groups.

Mock catalogue results show trends consistent with GAMA, although, as expected from the field LF comparison in Fig. A1, mock central galaxies tend to be offset to slightly lower luminosity than GAMA centrals, particularly in $\mathcal{M} 1$ groups.
Table 4. Schechter function fits (equation 6 with $\beta \equiv 1$ ) to the satellite galaxy LF for different galaxy samples as indicated. The final column gives the $\chi^{2}$ value and degrees of freedom $v$ of each fit.

\begin{tabular}{|c|c|c|c|c|c|}
\hline & $N_{\text {gal }}$ & $M^{*}$ & $\alpha$ & $\lg \phi_{S}^{*}$ & $\chi^{2} / v$ \\
\hline \multicolumn{6}{|c|}{ Mock all } \\
\hline $\mathcal{M} 1$ & 3273 & $-19.87 \pm 0.09$ & $-1.04 \pm 0.07$ & $-2.72 \pm 0.06$ & $3.3 / 9$ \\
\hline $\mathcal{M} 2$ & 4931 & $-20.34 \pm 0.07$ & $-0.65 \pm 0.09$ & $-3.02 \pm 0.03$ & $20.5 / 10$ \\
\hline $\mathcal{M} 3$ & 6037 & $-20.22 \pm 0.07$ & $-0.19 \pm 0.16$ & $-3.08 \pm 0.02$ & $21.4 / 11$ \\
\hline $\mathcal{M} 4$ & 6515 & $-20.44 \pm 0.06$ & $-0.44 \pm 0.11$ & $-3.17 \pm 0.03$ & $7.3 / 13$ \\
\hline \multicolumn{6}{|c|}{ GAMA all } \\
\hline $\mathcal{M} 1$ & 3579 & $-19.98 \pm 0.13$ & $-1.02 \pm 0.11$ & $-2.68 \pm 0.10$ & $9.7 / 8$ \\
\hline $\mathcal{M} 2$ & 5757 & $-20.32 \pm 0.08$ & $-0.73 \pm 0.10$ & $-3.01 \pm 0.04$ & $11.2 / 9$ \\
\hline $\mathcal{M} 3$ & 6014 & $-20.36 \pm 0.05$ & $-0.38 \pm 0.08$ & $-3.15 \pm 0.02$ & $18.9 / 10$ \\
\hline $\mathcal{M} 4$ & 6108 & $-20.83 \pm 0.05$ & $-0.68 \pm 0.08$ & $-3.38 \pm 0.02$ & $6.9 / 11$ \\
\hline \multicolumn{6}{|c|}{ GAMA blue } \\
\hline $\mathcal{M} 1$ & 2260 & $-20.28 \pm 0.20$ & $-1.30 \pm 0.09$ & $-3.09 \pm 0.11$ & $5.9 / 8$ \\
\hline $\mathcal{M} 2$ & 2837 & $-20.36 \pm 0.10$ & $-0.94 \pm 0.10$ & $-3.37 \pm 0.05$ & $10.2 / 9$ \\
\hline $\mathcal{M} 3$ & 2541 & $-20.35 \pm 0.08$ & $-0.56 \pm 0.10$ & $-3.55 \pm 0.03$ & $12.5 / 10$ \\
\hline $\mathcal{M} 4$ & 2271 & $-20.81 \pm 0.08$ & $-0.79 \pm 0.12$ & $-3.85 \pm 0.04$ & $9.9 / 11$ \\
\hline \multicolumn{6}{|c|}{ GAMA red } \\
\hline $\mathcal{M} 1$ & 1319 & $-19.69 \pm 0.16$ & $-0.49 \pm 0.18$ & $-3.06 \pm 0.10$ & $15.7 / 8$ \\
\hline $\mathcal{M} 2$ & 2920 & $-20.30 \pm 0.08$ & $-0.52 \pm 0.08$ & $-3.27 \pm 0.03$ & $6.8 / 9$ \\
\hline $\mathcal{M} 3$ & 3473 & $-20.47 \pm 0.06$ & $-0.43 \pm 0.08$ & $-3.40 \pm 0.02$ & $16.5 / 9$ \\
\hline $\mathcal{M} 4$ & 3837 & $-20.81 \pm 0.04$ & $-0.54 \pm 0.07$ & $-3.57 \pm 0.02$ & $8.9 / 11$ \\
\hline \multicolumn{6}{|c|}{ GAMA low-n } \\
\hline $\mathcal{M} 1$ & 2064 & $-19.47 \pm 0.10$ & $-1.02 \pm 0.11$ & $-2.79 \pm 0.08$ & $8.7 / 8$ \\
\hline $\mathcal{M} 2$ & 2551 & $-20.07 \pm 0.08$ & $-0.97 \pm 0.09$ & $-3.33 \pm 0.05$ & $4.7 / 9$ \\
\hline $\mathcal{M} 3$ & 2188 & $-20.06 \pm 0.05$ & $-0.54 \pm 0.09$ & $-3.51 \pm 0.02$ & $19.6 / 10$ \\
\hline $\mathcal{M} 4$ & 1928 & $-20.45 \pm 0.06$ & $-0.79 \pm 0.11$ & $-3.77 \pm 0.03$ & $11.7 / 10$ \\
\hline \multicolumn{6}{|c|}{ GAMA high-n } \\
\hline $\mathcal{M} 1$ & 1515 & $-20.22 \pm 0.25$ & $-0.74 \pm 0.19$ & $-3.10 \pm 0.13$ & $15.6 / 8$ \\
\hline $\mathcal{M} 2$ & 3206 & $-20.19 \pm 0.09$ & $-0.23 \pm 0.12$ & $-3.20 \pm 0.02$ & $9.9 / 9$ \\
\hline $\mathcal{M} 3$ & 3826 & $-20.42 \pm 0.06$ & $-0.25 \pm 0.10$ & $-3.35 \pm 0.02$ & $14.8 / 9$ \\
\hline $\mathcal{M} 4$ & 4180 & $-20.81 \pm 0.05$ & $-0.43 \pm 0.08$ & $-3.53 \pm 0.02$ & $6.9 / 11$ \\
\hline
\end{tabular}

\subsubsection{Colour and morphology dependence}

The LFs of colour- and Sérsic index-selected galaxies show similar behaviour. Within halo-mass bins, the central galaxy peak magnitude $M_{C}$ and satellite galaxy characteristic magnitude $M^{*}$ show remarkably little variation with galaxy colour (with the exception of $\mathcal{M} 1$ groups, in which blue galaxies are fainter in $M_{c}$, but brighter in $M^{*}$ ), whereas spheroidal galaxies tend to be brighter than disky galaxies. Relative to blue and disky galaxies, red and spheroidal galaxies are offset to a shallower (more positive) faint-end slope $\alpha$.

We see that red, and particularly spheroidal, galaxies dominate the central population, particularly at high halo masses. The spheroidal/disky ratio of centrals is larger than the red/blue ratio, particularly in higher-mass haloes.

\subsubsection{LF parameter trends with halo mass}

For central galaxies (lower-left panels of Fig. 7), we see that peak magnitude $M_{c}$ brightens systematically with halo mass. The width of the magnitude distribution $\sigma_{c}$ is largely independent of halo mass, although is broader in the lowest-mass haloes.

Within each satellite galaxy class we observe (lower-right panels of Fig. 7) a systematic and significant brightening of the characteristic magnitude $M^{*}$ with increasing halo mass. Any trends of faint-end slope $\alpha$ are less clear, although for most samples, galaxies in $\mathcal{M} 1$ haloes show the steepest faint-end slope. Mock galaxies show consistent trends with the 'GAMA all' sample.

For comparison, we also show, in the lower panels of Fig. 7, $\log$-normal and modified $(\beta \equiv 2)$ Schechter function fits to the cent- 

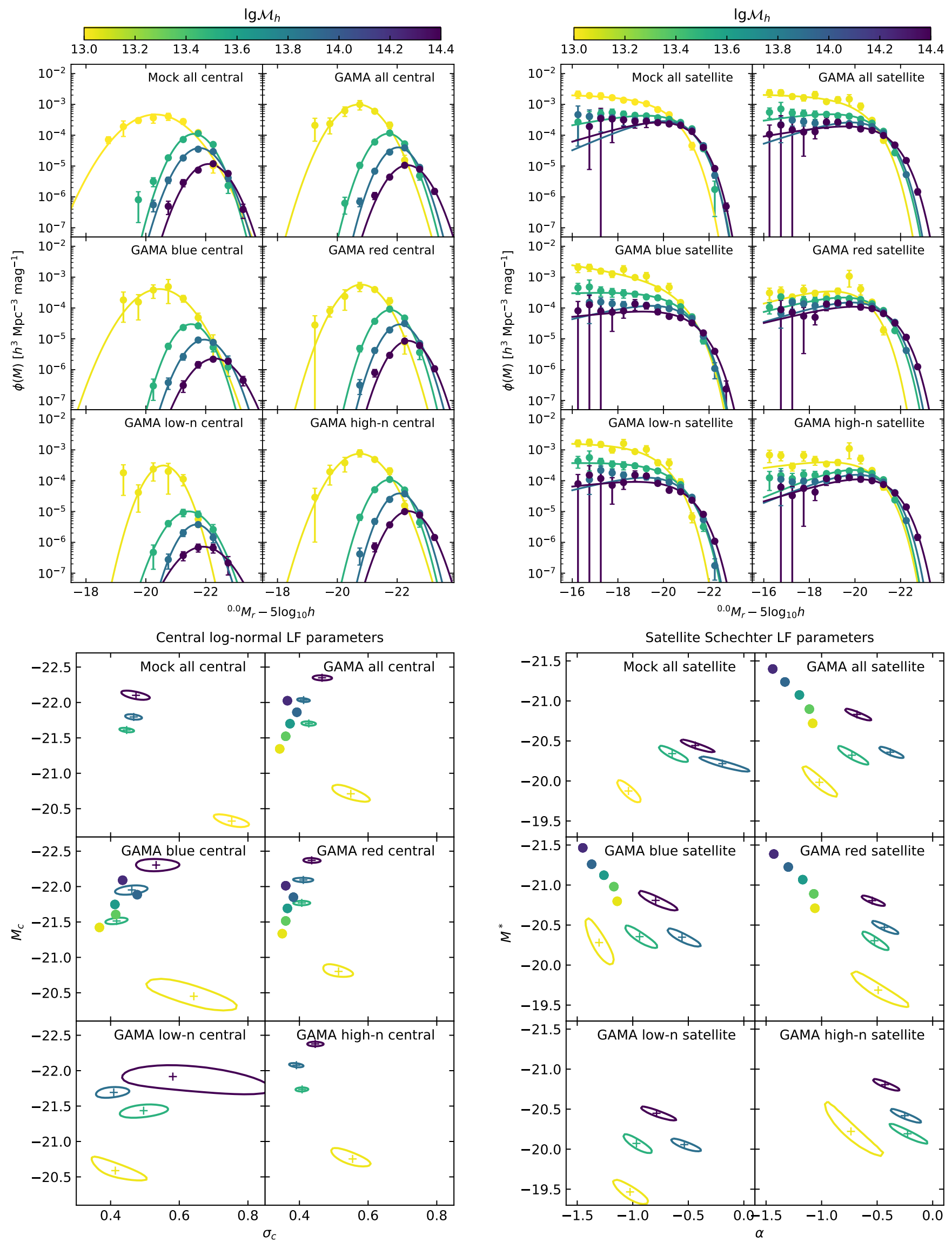

Figure 7. LFs colour-coded by halo mass, for central (left-hand panels) and satellite (right-hand panels) galaxy samples as labelled. Functional fits are lognormal for central galaxies and Schechter functions for satellites, with 1- $\sigma$ likelihood contours in lower-left and lower-right sets of panels, respectively. Filled circles in the lower panels show parameter fits from Yang et al. (2008). 
ral and satellite populations respectively from Yang et al. (2008) as filled circles (they do not split galaxies by morphology). We use parameter values from Table 1 of Yang et al. (2008) for their five halo mass bins within the range $13 \leq \lg \mathcal{M}_{h}<14$.4. Note that as well as the difference in satellite fitting function, Yang et al. (2008) $K$-correct to $z=0.1$ rather than $z=0.0$, and use a different colour cut, but one would nevertheless hope that trends with halo mass would be preserved.

For central galaxies, we observe consistent, but more pronounced, trends of $M_{C}$ with halo mass, cf. Yang et al. (2008). This difference could be explained by underestimated halo masses in Yang et al. (2007) single-galaxy groups (see fig. 6 of Davies et al. 2019), and so the Yang et al. (2008) low-mass bin likely mixes haloes of both low and high mass.

For satellite galaxies, the Yang et al. (2008) characteristic magnitudes $M^{*}$ and faint-end slopes $\alpha$ respectively are offset to significantly brighter and steeper values than ours, an effect attributable to the different choice of fitting function. Their observed trend of brightening $M^{*}$ with halo mass is consistent with ours. Contrary to our results, they see a clear steepening of faint-end slope $\alpha$ with increasing halo mass. One should note, however, that there is a hint in Yang et al. (2008, fig. 2) that the faint-end slope may be a little too shallow cf. their non-parametric estimates in lower-mass haloes.

\subsection{Group galaxy SMF}

Our SMF results, along with those from the L-GALAXIES SAM, and the EAGLE and IllustrisTNG simulations, are plotted in Fig. 8. Note that the relative normalisation of GAMA data and simulations is somewhat arbitrary, depending as it does on the halo mass limits. Log-normal and Schechter parameter fits for central and satellite galaxies respectively are shown in Fig. 9 and tabulated in Tables 5 and 6. We first discuss the observed SMF results for GAMA galaxies subdivided by central/satellite, colour and morphology, comparing with SDSS results from Yang et al. (2009). We then compare observed results with those from the L-GALAXIES SAM and simulations.

\subsubsection{Observed central versus satellite}

Fig. 8 plots the SMFs of central and satellite galaxies in the left- and right-hand panel sets, respectively. Unsurprisingly, central galaxies dominate at high stellar mass, while satellites dominate at low mass. As with the LFs, satellites become more dominant in highmass haloes due to the mass-richness correlation for groups.

On the whole, central galaxy SMFs are reasonably fit by lognormal functions (Table 5), although there are some statistically poor fits in the lower halo mass bins, due to a slight excess over the log-normal fit at lower masses. Schechter functions provide variable-quality fits to satellite SMFs (Table 6); in particular we observe a high-mass excess above the Schechter fit in higher-mass haloes. One can obtain a better fit by allowing the parameter $\beta$ in equation (6) to vary freely. However, the values of $\beta$ and $M^{*}$ are strongly correlated, and so parameter trends with halo mass are much harder to interpret, and also to compare with previous results. We thus choose to show only standard Schechter function fits $(\beta \equiv 1)$.

\subsubsection{Observed colour and morphology dependence}

At all halo masses, we see that red, and particularly spheroidal, galaxies dominate the central population. As with the LFs, the spheroidal/disky ratio of centrals is larger than the red/blue ratio. Our morphology-dependent results for low-mass haloes are qualitatively consistent with the field SMF results of Moffett et al. (2016), in which spheroidal and disky galaxies dominate at high and low stellar masses, respectively.

The SMFs of colour- and Sérsic index-selected galaxies show some subtle differences. For centrals, peak log-mass $M_{c}$ tends to be higher for red and spheroidal than for blue and disky galaxies; $M_{c}$ is particularly low for disky galaxies in $\mathcal{M} 1$ haloes. There are no significant differences in width parameter $\sigma_{c}$ apart from a broadening in $\mathcal{M} 1$ haloes, again particularly for disky galaxies. For satellites, spheroidal galaxies exhibit higher characteristic stellar mass $\mathcal{M}^{*}$ and shallower low-mass slope $\alpha$ than disky galaxies, whereas red and blue galaxies have more consistent SMF shapes, with the exception of steep low-mass slopes for blue galaxies in $\mathcal{M} 1$ haloes.

\subsubsection{Observed SMF parameter trends with halo mass}

For central galaxies (left panels of Fig. 9), we see that peak logmass $M_{C}$ increases systematically with halo mass, and is $\sim 0.2 \mathrm{dex}$ higher for red and spheroidal galaxies than their blue and disky counterparts. The width of the mass distribution $\sigma_{c}$ tends to increase for lower halo masses, particularly for disky galaxies, whose M1 likelihood contour lies well off the bottom-right limits of the plot.

Within each satellite galaxy class we observe (right panels of Fig. 9) a systematic increase in characteristic mass $M^{*}$ with increasing halo mass. There is little significant trend of low-mass slope $\alpha$ with halo mass, except that it is much steeper for blue and disky galaxies in $\mathcal{M} 1$ haloes.

For comparison, we also show log-normal and modified $(\beta \equiv$ 2) Schechter function fits to the central and satellite populations, respectively, from Yang et al. (2009) as filled circles (they do not split galaxies by morphology). We use parameter values from Table 4 of Yang et al. (2009) for their five halo mass bins within the range $13 \leq \lg \mathcal{M}_{h}<14.4$. Note that the Yang et al. (2009) colour-cut is different to ours, but trends with halo mass should not be strongly affected. As with the LFs, the Yang et al. (2009) satellite SMF parameters are offset to brighter and steeper values than ours, due to the different choice of power within the Schechter function exponential. We observe consistent trends in peak and characteristic stellar mass with halo mass for central and satellite galaxies, respectively, although our halo mass dependence is slightly stronger. Again, this is likely to be due to the Yang et al. (2009) low-mass bins containing a range of halo masses. Yang et al. (2009) find narrower log-normal fits to centrals, possibly reflecting their narrower bins in halo mass. Unlike Yang et al. (2009), we do not observe a systematic steepening of satellite low-mass slope $\alpha$ with halo mass. Again, we note (Yang et al. 2009, fig. 4) that their low-mass slopes in low-mass haloes may be a little too shallow.

\subsubsection{Comparison of GAMA and simulated SMFs}

For central galaxies, L-GALAXIES and EAGLE show log-normal parameters and trends consistent with observations, with the caveat that the small volume of the EAGLE simulation $(27 \times$ smaller than TNG-300), means that there are very few massive galaxies, hence 

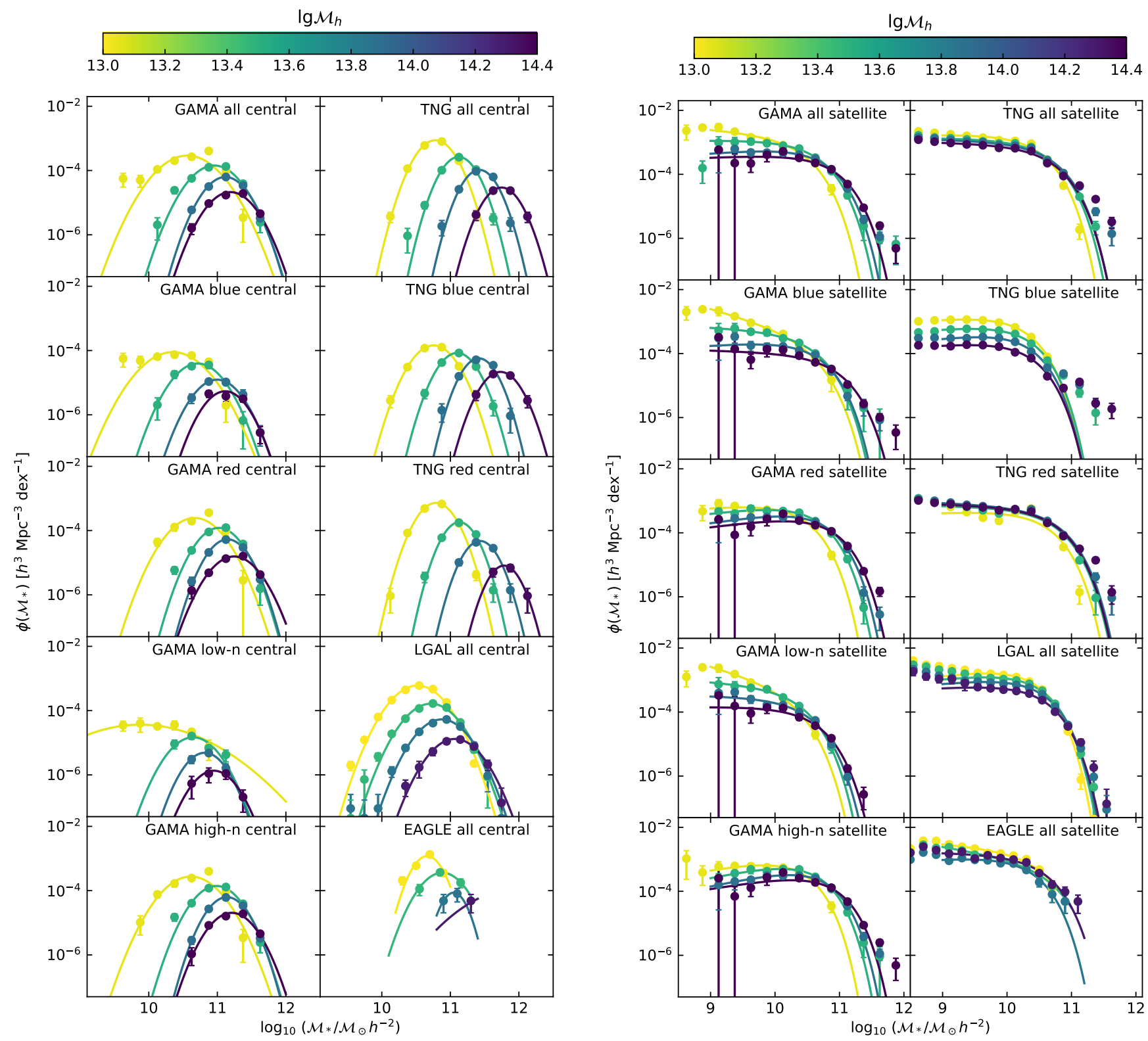

Figure 8. SMFs colour-coded by halo mass, for galaxy samples as labelled in each panel. Central and satellite galaxies are shown in left- and right-hand panel sets, and fitted with log-normal and Schechter functions, respectively.

the central fits are poorly constrained. IllustrisTNG groups, however, host much more massive central galaxies than the observations, and show an enhanced dependence of $M_{C}$ on halo mass. Central galaxy stellar masses in IllustrisTNG thus seem to be both too high and also over-dependent on halo mass.

For satellite galaxies, the functional fits to the large-volume L-GALAXIES and IllustrisTNG SMFs are often statistically very poor, with some reduced $\chi^{2}$ values in excess of 10 . This is partly due to the large numbers of galaxies in these simulations giving rise to very high signal-to-noise measurements, but is also due to the fact that Schechter fits drop too steeply at the high mass end in massive haloes. The discrepancy is even worse with the $\beta \equiv$ 2 modified Schechter functions used by Yang et al. (2009). One can get a slightly better fit to the IllustrisTNG results by allowing $\beta$ to vary freely, but the $\chi^{2}$ values are still poor in many cases. In particular, no value of $\beta$ can match the very shallow high-mass shape of the IllustrisTNG blue satellite SMF. The L-GALAXIES SMFs clearly favour a double Schechter function, with a steeper slope below mass $\lg \mathcal{M}_{*} \approx 9.5$.

The Schechter fits to satellite galaxies show much smaller shape variation with halo mass than the observations. SDSS and GAMA observe an increase in characteristic mass of $\Delta \lg \mathcal{M}_{*} \approx 0.4$ and 0.5 dex respectively from $\mathcal{M} 1-\mathcal{M} 4$ haloes, whereas the simulations yield $\Delta \lg \mathcal{M}_{*}<0.3 \mathrm{dex}$. The IllustrisTNG and L-GALAXIES SMFs show only a very small steepening of low-mass slope $\alpha$ with halo mass; EAGLE shows larger variation in both $\lg \mathcal{M}^{*}$ and $\alpha$, but no systematic trends with halo mass. We caution that the likelihood contours for simulated satellite galaxies in Fig. 9 arise from functional fits that are in some cases statistically very poor. It can be seen visually in Fig. 8 that the SMF shapes of low to moderate mass satellite galaxies in the L-GALAXIES SAM and the simulations appear to be almost independent of their host halo mass. Only 

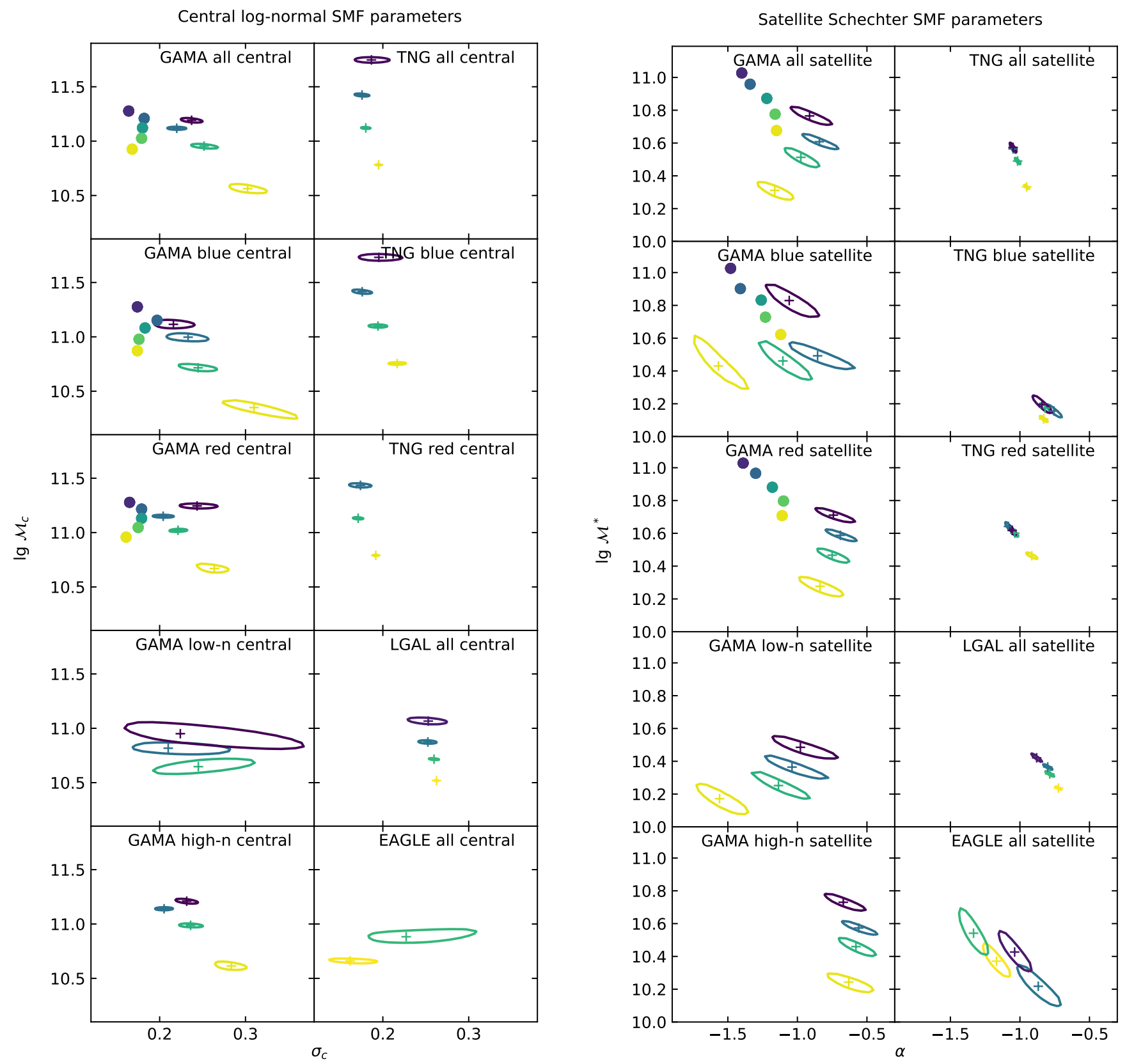

Figure 9. 1- $\sigma$ likelihood contours for log-normal fits to central galaxies (left) and Schechter function parameter fits to satellite SMFs (right), colour-coded by halo mass using the same scheme as Fig. 8. Filled circles show parameter fits from Yang et al. (2009).

for the most massive satellites $\left(\lg \mathcal{M}_{*} \gtrsim 10.5\right)$, does their abundance increases significantly with halo mass.

\subsection{LF and SMF Evolution}

LFs and SMFs determined in halo mass bins and redshift slices ${ }^{6}$ are plotted in Fig. 10 (upper and lower set of panels, respectively), and show a consistent picture. Symbols show LFs/SMFs in group mass bins (rows) and for different galaxy types (columns), as labelled.

\footnotetext{
6 We note that the EAGLE halo mass function exhibits negligible evolution over the redshift range shown, and so do not expect GAMA halo masses to evolve significantly.
}

For comparison purposes, lines show LFs/SMFs of field galaxies (i.e. whether grouped or not) of corresponding type, renormalized to the number of grouped galaxies in each panel (i.e. of given type and environment, but summing over redshift bins). For all types of GAMA galaxies, the field LFs show evidence for minor fading in $M^{*}$ since redshift $z \approx 0.3$. The field SMFs show little sign of evolution, apart from a paucity of the most massive galaxies at low redshift.

In the first column of both panel sets (all galaxies), we see that the relative number densities of luminous $\left(M_{r} \lesssim-21 \mathrm{mag}\right)$ and massive $\left(\lg M_{*} \gtrsim 10.5\right)$ grouped galaxies are enhanced over the field in both group environments at redshifts $z \gtrsim 0.1$. In the only redshift bin $(z<0.1)$ in which faint $\left(M_{r} \gtrsim-18 \mathrm{mag}\right)$ galaxies are visible, they are relatively less abundant than in the field. Dwarf 
Table 5. Log-normal fits (equation 5) to the central galaxy SMF for different galaxy samples as indicated. The column headed $\chi^{2} / v$ gives the $\chi^{2}$ value and degrees of freedom for the functional fit.

\begin{tabular}{|c|c|c|c|c|c|}
\hline & $N_{\text {gal }}$ & $\lg \mathcal{M}_{c}$ & $\sigma_{c}$ & $\lg \phi_{c}^{*}$ & $\chi^{2} / v$ \\
\hline \multicolumn{6}{|c|}{ GAMA all } \\
\hline $\mathcal{M} 1$ & 684 & $10.56 \pm 0.03$ & $0.30 \pm 0.02$ & $-3.54 \pm 0.04$ & $28.5 / 7$ \\
\hline $\mathcal{M} 2$ & 811 & $10.95 \pm 0.01$ & $0.25 \pm 0.01$ & $-3.84 \pm 0.04$ & $13.2 / 5$ \\
\hline $\mathcal{M} 3$ & 644 & $11.12 \pm 0.01$ & $0.22 \pm 0.01$ & $-4.20 \pm 0.03$ & $3.5 / 3$ \\
\hline $\mathcal{M} 4$ & 369 & $11.19 \pm 0.01$ & $0.24 \pm 0.01$ & $-4.67 \pm 0.03$ & $9.5 / 3$ \\
\hline \multicolumn{6}{|c|}{ GAMA blue } \\
\hline $\mathcal{M} 1$ & 200 & $10.35 \pm 0.06$ & $0.31 \pm 0.03$ & $-4.05 \pm 0.05$ & $16.1 / 7$ \\
\hline $\mathcal{M} 2$ & 202 & $10.72 \pm 0.02$ & $0.24 \pm 0.02$ & $-4.40 \pm 0.06$ & $2.6 / 4$ \\
\hline $\mathcal{M} 3$ & 143 & $11.00 \pm 0.03$ & $0.23 \pm 0.02$ & $-4.91 \pm 0.05$ & $0.7 / 3$ \\
\hline $\mathcal{M} 4$ & 80 & $11.12 \pm 0.03$ & $0.22 \pm 0.02$ & $-5.27 \pm 0.08$ & $6.1 / 3$ \\
\hline \multicolumn{6}{|c|}{ GAMA red } \\
\hline $\mathcal{M} 1$ & 484 & $10.67 \pm 0.03$ & $0.26 \pm 0.01$ & $-3.61 \pm 0.05$ & $17.0 / 4$ \\
\hline $\mathcal{M} 2$ & 609 & $11.02 \pm 0.01$ & $0.22 \pm 0.01$ & $-3.92 \pm 0.04$ & $11.9 / 4$ \\
\hline $\mathcal{M} 3$ & 501 & $11.15 \pm 0.01$ & $0.20 \pm 0.01$ & $-4.27 \pm 0.03$ & $0.9 / 2$ \\
\hline $\mathcal{M} 4$ & 289 & $11.24 \pm 0.02$ & $0.24 \pm 0.02$ & $-4.81 \pm 0.04$ & $3.3 / 2$ \\
\hline \multicolumn{6}{|c|}{ GAMA low-n } \\
\hline $\mathcal{M} 1$ & 97 & $9.88 \pm 0.25$ & $0.64 \pm 0.18$ & $-4.44 \pm 0.06$ & $1.9 / 4$ \\
\hline $\mathcal{M} 2$ & 73 & $10.65 \pm 0.05$ & $0.25 \pm 0.04$ & $-4.83 \pm 0.09$ & $3.3 / 2$ \\
\hline $\mathcal{M} 3$ & 41 & $10.82 \pm 0.04$ & $0.21 \pm 0.04$ & $-5.31 \pm 0.07$ & $0.2 / 1$ \\
\hline $\mathcal{M} 4$ & 20 & $10.95 \pm 0.07$ & $0.22 \pm 0.06$ & $-5.87 \pm 0.14$ & $0.2 / 1$ \\
\hline \multicolumn{6}{|c|}{ GAMA high-n } \\
\hline $\mathcal{M} 1$ & 587 & $10.61 \pm 0.03$ & $0.28 \pm 0.01$ & $-3.56 \pm 0.04$ & $20.0 / 6$ \\
\hline $\mathcal{M} 2$ & 738 & $10.99 \pm 0.01$ & $0.24 \pm 0.01$ & $-3.85 \pm 0.04$ & $10.7 / 5$ \\
\hline $\mathcal{M} 3$ & 603 & $11.14 \pm 0.01$ & $0.21 \pm 0.01$ & $-4.20 \pm 0.03$ & $0.6 / 2$ \\
\hline $\mathcal{M} 4$ & 349 & $11.21 \pm 0.02$ & $0.23 \pm 0.01$ & $-4.69 \pm 0.03$ & $9.8 / 3$ \\
\hline \multicolumn{6}{|c|}{ TNG all } \\
\hline $\mathcal{M} 1$ & 3713 & $10.78 \pm 0.00$ & $0.20 \pm 0.00$ & $-3.06 \pm 0.01$ & $20.9 / 3$ \\
\hline $\mathcal{M} 2$ & 1039 & $11.12 \pm 0.01$ & $0.18 \pm 0.00$ & $-3.58 \pm 0.02$ & $8.0 / 3$ \\
\hline $\mathcal{M} 3$ & 405 & $11.42 \pm 0.01$ & $0.18 \pm 0.01$ & $-3.98 \pm 0.03$ & $7.1 / 2$ \\
\hline $\mathcal{M} 4$ & 119 & $11.75 \pm 0.02$ & $0.19 \pm 0.01$ & $-4.53 \pm 0.05$ & $0.0 / 1$ \\
\hline \multicolumn{6}{|c|}{$\begin{array}{l}\text { TNG blue } \\
\end{array}$} \\
\hline $\mathcal{M} 1$ & 671 & $10.75 \pm 0.01$ & $0.22 \pm 0.01$ & $-3.84 \pm 0.02$ & $0.6 / 2$ \\
\hline $\mathcal{M} 2$ & 355 & $11.10 \pm 0.01$ & $0.19 \pm 0.01$ & $-4.07 \pm 0.03$ & $1.0 / 3$ \\
\hline $\mathcal{M} 3$ & 225 & $11.41 \pm 0.01$ & $0.18 \pm 0.01$ & $-4.24 \pm 0.04$ & $7.0 / 2$ \\
\hline $\mathcal{M} 4$ & 91 & $11.73 \pm 0.02$ & $0.20 \pm 0.02$ & $-4.66 \pm 0.06$ & $0.0 / 1$ \\
\hline \multicolumn{6}{|c|}{ TNG red } \\
\hline $\mathcal{M} 1$ & 3042 & $10.79 \pm 0.00$ & $0.19 \pm 0.00$ & $-3.14 \pm 0.01$ & $13.6 / 3$ \\
\hline $\mathcal{M} 2$ & 684 & $11.13 \pm 0.01$ & $0.17 \pm 0.00$ & $-3.74 \pm 0.02$ & $8.4 / 3$ \\
\hline $\mathcal{M} 3$ & 180 & $11.43 \pm 0.01$ & $0.17 \pm 0.01$ & $-4.32 \pm 0.04$ & $1.2 / 2$ \\
\hline $\mathcal{M} 4$ & 28 & $11.78 \pm 9.99$ & $0.16 \pm 9.99$ & $-5.09 \pm 9.99$ & $0.0 / 0$ \\
\hline \multicolumn{6}{|c|}{$\begin{array}{l}\text { LGAL all } \\
\end{array}$} \\
\hline $\mathcal{M} 1$ & 44636 & $10.52 \pm 0.00$ & $0.26 \pm 0.00$ & $-3.22 \pm 0.00$ & $70.1 / 7$ \\
\hline $\mathcal{M} 2$ & 11893 & $10.72 \pm 0.01$ & $0.26 \pm 0.00$ & $-3.78 \pm 0.01$ & $20.1 / 8$ \\
\hline $\mathcal{M} 3$ & 3660 & $10.87 \pm 0.01$ & $0.25 \pm 0.01$ & $-4.27 \pm 0.02$ & $6.7 / 9$ \\
\hline $\mathcal{M} 4$ & 910 & $11.07 \pm 0.02$ & $0.25 \pm 0.02$ & $-4.88 \pm 0.04$ & $2.2 / 5$ \\
\hline \multicolumn{6}{|c|}{ EAGLE all } \\
\hline $\mathcal{M} 1$ & 155 & $10.66 \pm 0.02$ & $0.16 \pm 0.02$ & $-2.93 \pm 0.06$ & $7.3 / 1$ \\
\hline $\mathcal{M} 2$ & 42 & $10.88 \pm 0.04$ & $0.23 \pm 0.04$ & $-3.44 \pm 0.10$ & $0.4 / 1$ \\
\hline $\mathcal{M} 3$ & 9 & $11.05 \pm 9.99$ & $0.14 \pm 9.99$ & $-4.06 \pm 9.99$ & $0.0 / 0$ \\
\hline $\mathcal{M} 4$ & 5 & $12.00 \pm 9.99$ & $0.49 \pm 9.99$ & $-3.93 \pm 9.99$ & $0.3 / 0$ \\
\hline
\end{tabular}

galaxies when selected by mass $\left(\lg M_{*} \lesssim 9.5\right)$ are more consistent with the field, but still slightly suppressed. Overall, the number density of grouped galaxies at low redshift $(z<0.1)$ is below that in the field, given our renormalization across all redshifts.

Blue and disky galaxies (second and fourth columns) show similar behaviour to the general population. Blue galaxies show a slightly larger excess than disky galaxies over their respective field populations at the bright/high-mass end at redshifts $z>0.1$.

Only red galaxies (third column) show a number density consistent with the renormalized field at low luminosities/masses and
Table 6. Schechter function fits (equation 6 with $\beta \equiv 1$ ) to the satellite galaxy SMF for different galaxy samples as indicated. The column headed $\chi^{2} / v$ gives the $\chi^{2}$ value and degrees of freedom for the functional fit.

\begin{tabular}{|c|c|c|c|c|c|}
\hline & $N_{\mathrm{gal}}$ & $\lg \mathcal{M}^{*}$ & $\alpha$ & $\lg \phi_{s}^{*}$ & $\chi^{2} / v$ \\
\hline \multicolumn{6}{|c|}{ GAMA all } \\
\hline $\mathcal{M} 1$ & 1882 & $10.31 \pm 0.04$ & $-1.16 \pm 0.09$ & $-3.17 \pm 0.07$ & $7.4 / 7$ \\
\hline M2 & 3183 & $10.51 \pm 0.04$ & $-0.98 \pm 0.09$ & $-3.27 \pm 0.05$ & $5.2 / 9$ \\
\hline M3 & 2996 & $10.61 \pm 0.03$ & $-0.84 \pm 0.09$ & $-3.45 \pm 0.05$ & $9.0 / 9$ \\
\hline M4 & 3147 & $10.77 \pm 0.04$ & $-0.91 \pm 0.11$ & $-3.68 \pm 0.06$ & $22.5 / 9$ \\
\hline \multicolumn{6}{|c|}{ GAMA blue } \\
\hline M1 & 895 & $10.43 \pm 0.11$ & $-1.57 \pm 0.14$ & $-3.77 \pm 0.19$ & $1.7 / 5$ \\
\hline M2 & 1125 & $10.46 \pm 0.09$ & $-1.10 \pm 0.14$ & $-3.70 \pm 0.11$ & $4.5 / 9$ \\
\hline M3 & 836 & $10.49 \pm 0.06$ & $-0.85 \pm 0.17$ & $-3.89 \pm 0.08$ & $11.3 / 9$ \\
\hline M4 & 736 & $10.83 \pm 0.07$ & $-1.06 \pm 0.13$ & $-4.37 \pm 0.10$ & $15.7 / 9$ \\
\hline \multicolumn{6}{|c|}{ GAMA red } \\
\hline $\mathcal{M} 1$ & 987 & $10.28 \pm 0.04$ & $-0.84 \pm 0.11$ & $-3.37 \pm 0.06$ & $6.7 / 7$ \\
\hline M2 & 2058 & $10.47 \pm 0.03$ & $-0.75 \pm 0.08$ & $-3.39 \pm 0.04$ & $3.7 / 8$ \\
\hline M3 & 2160 & $10.59 \pm 0.03$ & $-0.69 \pm 0.08$ & $-3.56 \pm 0.04$ & $5.1 / 8$ \\
\hline M4 & 2411 & $10.71 \pm 0.03$ & $-0.74 \pm 0.10$ & $-3.74 \pm 0.05$ & $13.5 / 9$ \\
\hline \multicolumn{6}{|c|}{ GAMA low-n } \\
\hline $\mathcal{M} 1$ & 770 & $10.17 \pm 0.07$ & $-1.56 \pm 0.13$ & $-3.57 \pm 0.12$ & $1.5 / 5$ \\
\hline M2 & 923 & $10.25 \pm 0.06$ & $-1.14 \pm 0.15$ & $-3.58 \pm 0.09$ & $1.8 / 6$ \\
\hline $\mathcal{M} 3$ & 617 & $10.36 \pm 0.05$ & $-1.04 \pm 0.16$ & $-3.91 \pm 0.08$ & $4.5 / 6$ \\
\hline M4 & 513 & $10.48 \pm 0.05$ & $-0.98 \pm 0.16$ & $-4.17 \pm 0.07$ & $6.1 / 7$ \\
\hline \multicolumn{6}{|c|}{ GAMA high-n } \\
\hline $\mathcal{M} 1$ & 1112 & $10.24 \pm 0.04$ & $-0.63 \pm 0.12$ & $-3.23 \pm 0.05$ & $3.6 / 7$ \\
\hline$M 2$ & 2260 & $10.46 \pm 0.04$ & $-0.58 \pm 0.09$ & $-3.32 \pm 0.04$ & $7.1 / 9$ \\
\hline M3 & 2379 & $10.57 \pm 0.03$ & $-0.56 \pm 0.09$ & $-3.51 \pm 0.04$ & $8.4 / 9$ \\
\hline M4 & 2634 & $10.73 \pm 0.04$ & $-0.67 \pm 0.10$ & $-3.72 \pm 0.05$ & $24.2 / 9$ \\
\hline & & & TNG all & & \\
\hline $\mathcal{M} 1$ & 26857 & $10.33 \pm 0.01$ & $-0.95 \pm 0.01$ & $-3.06 \pm 0.01$ & $274.5 / 6$ \\
\hline$M 2$ & 20870 & $10.49 \pm 0.01$ & $-1.02 \pm 0.01$ & $-3.26 \pm 0.02$ & $119.9 / 7$ \\
\hline M3 & 18650 & $10.56 \pm 0.02$ & $-1.05 \pm 0.02$ & $-3.36 \pm 0.02$ & $119.1 / 8$ \\
\hline M4 & 15349 & $10.57 \pm 0.02$ & $-1.05 \pm 0.02$ & $-3.45 \pm 0.02$ & $137.4 / 8$ \\
\hline \multicolumn{6}{|c|}{ TNG blue } \\
\hline $\mathcal{M} 1$ & 16161 & $10.11 \pm 0.01$ & $-0.83 \pm 0.02$ & $-3.10 \pm 0.01$ & $2.6 / 6$ \\
\hline $\mathcal{M} 2$ & 8377 & $10.17 \pm 0.02$ & $-0.82 \pm 0.03$ & $-3.38 \pm 0.02$ & $39.0 / 7$ \\
\hline M3 & 4901 & $10.16 \pm 0.03$ & $-0.76 \pm 0.04$ & $-3.60 \pm 0.03$ & $69.5 / 8$ \\
\hline M4 & 2789 & $10.20 \pm 0.04$ & $-0.84 \pm 0.05$ & $-3.90 \pm 0.04$ & $50.5 / 8$ \\
\hline \multicolumn{6}{|c|}{ TNG red } \\
\hline M1 & 10696 & $10.46 \pm 9.99$ & $-0.91 \pm 9.99$ & $-3.61 \pm 9.99$ & $1074.3 / 6$ \\
\hline M2 & 12493 & $10.60 \pm 0.02$ & $-1.04 \pm 0.02$ & $-3.58 \pm 0.02$ & $308.6 / 7$ \\
\hline M3 & 13749 & $10.64 \pm 0.02$ & $-1.08 \pm 0.02$ & $-3.56 \pm 0.02$ & $178.1 / 8$ \\
\hline M4 & 12560 & $10.62 \pm 0.02$ & $-1.06 \pm 0.02$ & $-3.56 \pm 0.02$ & $131.9 / 8$ \\
\hline \multicolumn{6}{|c|}{ LGAL all } \\
\hline $\mathcal{M} 1$ & 156902 & $10.24 \pm 0.01$ & $-0.72 \pm 0.02$ & $-2.88 \pm 0.01$ & $33.6 / 9$ \\
\hline $\mathcal{M} 2$ & 122491 & $10.32 \pm 0.01$ & $-0.79 \pm 0.02$ & $-3.04 \pm 0.01$ & $24.2 / 9$ \\
\hline M3 & 90289 & $10.36 \pm 0.01$ & $-0.80 \pm 0.02$ & $-3.20 \pm 0.01$ & $25.3 / 10$ \\
\hline M4 & 60075 & $10.42 \pm 0.02$ & $-0.88 \pm 0.03$ & $-3.44 \pm 0.02$ & $27.5 / 10$ \\
\hline \multicolumn{6}{|c|}{ EAGLE all } \\
\hline $\mathcal{M} 1$ & 1457 & $10.37 \pm 0.07$ & $-1.17 \pm 0.07$ & $-3.11 \pm 0.08$ & $9.9 / 6$ \\
\hline $\mathcal{M} 2$ & 1027 & $10.54 \pm 0.10$ & $-1.33 \pm 0.07$ & $-3.48 \pm 0.12$ & $3.6 / 7$ \\
\hline M3 & 635 & $10.22 \pm 0.09$ & $-0.87 \pm 0.11$ & $-3.20 \pm 0.09$ & $4.9 / 8$ \\
\hline M4 & 945 & $10.43 \pm 0.09$ & $-1.04 \pm 0.09$ & $-3.21 \pm 0.09$ & $13.7 / 8$ \\
\hline
\end{tabular}

low redshift. This is to be expected if star formation is quenched in the infalling galaxies, leading to an increased abundance of red galaxies. Low-luminosity and low-mass red galaxies in $\mathcal{M} 4$ groups at $0.1<z<0.2$ show a slight excess relative to the field.

Spheroidal galaxies (final column) show similar behaviour to the overall population, with no low-luminosity/mass, low-redshift, excess, when compared with red galaxies. This suggests that the quenching process has not yet had time to fully transform the morphological appearance of infalling galaxies.

Note that, within each halo mass bin, the mean halo mass 

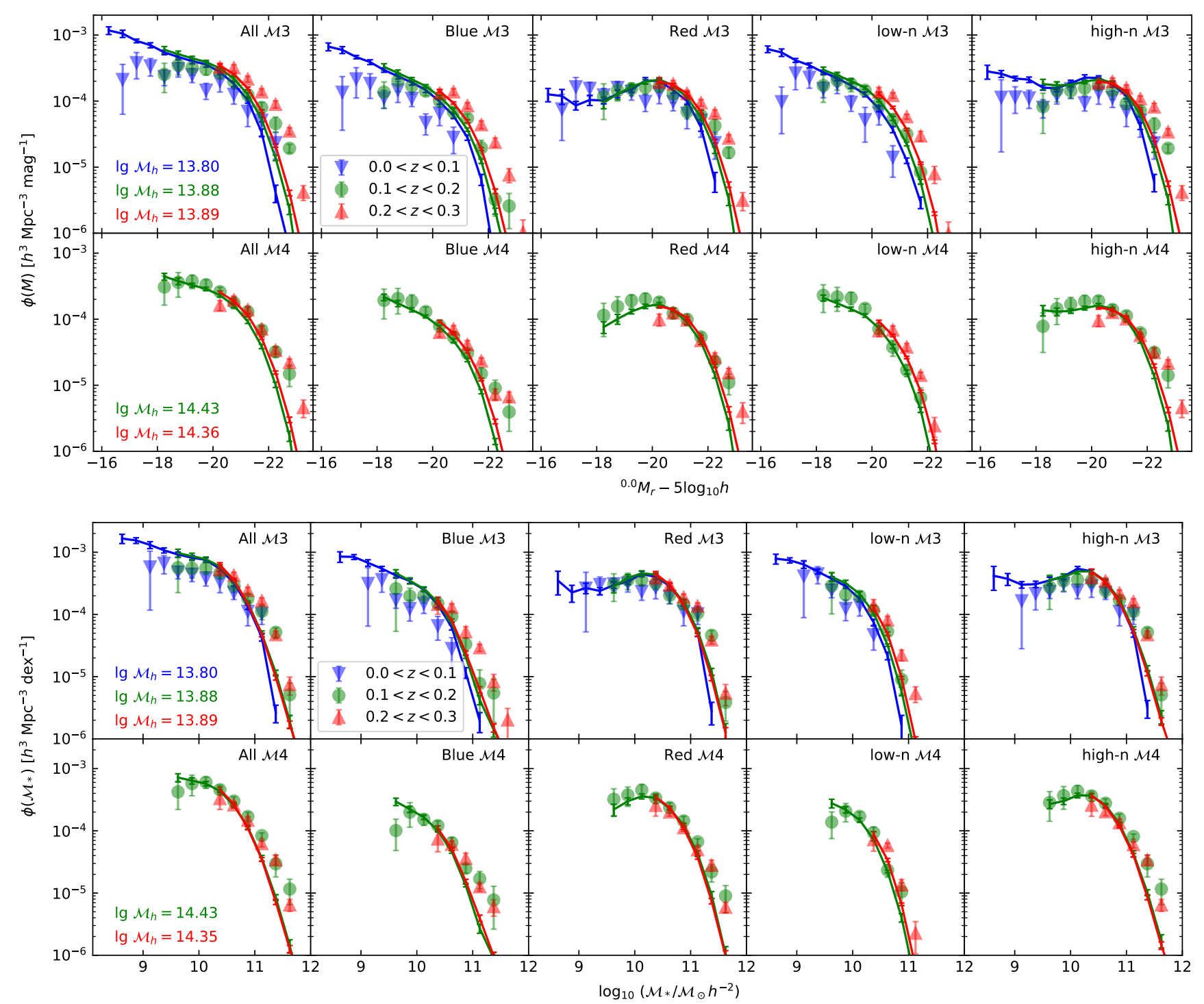

Figure 10. Grouped galaxy LFs and SMFs are shown as symbols in the upper and lower set of panels, respectively. Within each set of panels, galaxies are subdivided by halo mass (rows) and galaxy type (columns), with colour coding and symbol shape indicating redshift range. The numbers in the left-most panels indicate the mean halo mass within each redshift bin, showing that, within halo mass bins, there is relatively little dependence of halo mass on redshift. For comparison purposes, lines show the LFs or SMFs of field galaxies, whether grouped or not, renormalised to the number of grouped galaxies in each panel. No evolution corrections are applied to the LFs.

shows only a weak dependence on redshift, as indicated by the numbers in the left-most panels of Fig. 10. We thus believe that the changes in group LF or SMF with redshift are primarily due to evolution rather than varying host halo mass. As a caveat, however, we note that due to the flux-limited nature of the GAMA sample, groups of fixed mass have more detected members when observed at lower redshift (Fig. 2), which may have some effect on the apparent evolution measured. However, it seems unlikely that this would result in the differences in behaviour seen for blue and red galaxies.

\section{CONCLUSIONS AND DISCUSSION}

In this work we have presented the $r$-band LF and the SMF for galaxies in the GAMA group catalogue $\left(\mathrm{G}^{3} \mathrm{Cv} 9\right)$ separated into central and satellite, and divided by colour and morphology. The group catalogue was divided into four mass bins $\mathcal{M} 1-4$ covering $\lg \mathcal{M}_{h}=12-15.2$ to explore the dependency of the LF and SMF on group mass, and later into three redshift bins below $z=0.3$ to investigate LF/SMF evolution.

On subdividing galaxies into central and satellite populations, we see that centrals are always luminous and massive, with fainter $\left(M_{r} \gtrsim-19 \mathrm{mag}\right)$ galaxies being exclusively satellites, particularly in higher-mass haloes. We note that the only (indirect) use of mass in defining a GAMA central galaxy is the choice of the brighter of the two galaxies that remain after iteratively rejecting galaxies furthest from the group centre-of-light (see R11 sec 4.2.1). This contrasts with, for example, Yang et al. (2008, 2009); Knobel et al. (2015), who define the central galaxy as the most luminous or massive in the group. 
Due to the correlation between group mass and richness, satellite galaxies become more and more numerically dominant in higher-mass groups. Note that the definition of GAMA groups is very different to that of Yang et al. (2007), whose group definition includes those comprising single galaxies.

When subdividing the galaxy population by colour and Sérsic index, we find that red and, in particular, spheroidal galaxies dominate at high luminosity and mass; blue and disky galaxies dominate, or at least contribute roughly equally, at low luminosity and mass. The fraction of galaxies classified as red or spheroidal increases with increasing halo mass, consistent with the findings of Davies et al. (2019).

We next summarize our results separately for central and satellite galaxies, and for low-redshift evolution, and then put these findings in context.

\subsection{Central galaxy LF and SMF}

The observed central galaxy LF and SMF are well-fit by log-normal functions, with peak luminosity and mass increasing systematically with host halo mass. These trends are consistent with the SDSS measurements by Yang et al. (2008, 2009), except that we observe a much stronger dependence: comparing $\mathcal{M} 4$ and $\mathcal{M} 1$ haloes, we observe $\Delta M_{C} \approx 1.6 \mathrm{mag}$ or 0.6 dex for the LF and SMF respectively, compared with $\approx 0.7 \mathrm{mag}$ and $\approx 0.4 \mathrm{dex}$ from SDSS. These differences can be understood if the SDSS halo masses are underestimated for single-member groups, as indicated by the comparison in fig. 6 of Davies et al. (2019). This would imply that the lowerhalo mass bins in Yang et al. $(2008,2009)$ actually contain haloes with a wide range of masses.

The luminosity and mass distributions tend to be broader in the $\mathcal{M} 1$ bin, which covers the widest range of halo masses. The narrower log-normal fits from Yang et al. $(2008,2009)$ likely reflect their use of narrower bins in halo mass.

GAMA mock central LFs show consistent trends with the observations, albeit offset to slightly lower luminosities. The L-GALAXIES SAM shows central SMF parameters consistent with GAMA, whereas the IllustrisTNG simulation yields peak masses both higher than ours, and with an even more enhanced dependence on halo mass $\left(\Delta M_{C} \approx 1.0 \mathrm{dex}\right)$. This suggests that the IllustrisTNG central galaxy stellar masses are overly-dependent on halo mass. The small volume of the EAGLE simulation provides only weak constraints on the halo-mass dependence of the central SMF.

\subsection{Satellite galaxy LF and SMF}

The observed satellite galaxy LF and SMF are reasonably wellfit by standard Schechter functions, with characteristic luminosity and mass increasing systematically with host halo mass, in agreement with all previous studies. Faint-end and low-mass slopes of the satellite LF and SMF, respectively, show little systematic correlation with halo mass, except that galaxies in the lowest mass haloes tend to have the most steeply-rising slopes. This is in disagreement with some previous group LF/SMF results such as Yang et al. (2008, 2009); Robotham et al. (2010); Zandivarez \& Martínez (2011), but in agreement with Phillipps et al. (1998), who find that dwarfs are more common in lower density environments. Reddick et al. (2013, fig. 15) see no clear dependence of CSMF slope on halo mass for their SDSS group catalogue. Such discrepancies are likely to arise due to the inability of Schechter-like functions to accurately match the observed LF shape over a wide range of luminosities: the faint-end slope is often more strongly constrained by high signalto-noise measurements around $L^{*}$ than by the faintest galaxies in the sample. The same argument applies when fitting the SMF.

We note that the dependence of the faint- or low-mass slope on local density as estimated by galaxy counts in cylinders or spheres is similarly ambiguous, with some authors (e.g. Xia et al. 2006; Peng et al. 2010) finding a steepening faint-end/low-mass slope in denser environments, at least for red galaxies, while others (e.g. Croton et al. 2005; Hoyle et al. 2005; McNaught-Roberts et al. 2014; Mortlock et al. 2015) find no such dependence.

Turning now to the GAMA mock catalogues, the LFs have a higher abundance of faint satellite galaxies in massive haloes compared with GAMA data. It is beyond the scope of this paper to explore the physical reasons for this, but we do compare predictions of two more recent SAMs with GAMA data in Riggs et al. (in prep.). Standard Schechter functions systematically underestimate the faint-end of the LF in all but $\mathcal{M} 1$ mock groups, and so the resulting parameter fits should be treated with due caution.

The SMF shape in all simulations is generally independent of halo mass at low stellar masses, $\lg \mathcal{M}_{*} \lesssim 10.5$, whereas the GAMA low-mass slope is steeper in $\mathcal{M} 1$ groups. Only at high stellar masses, $\lg \mathcal{M}_{*} \gtrsim 10.5$, do the simulations reveal an increasing number density in higher-mass haloes. Schechter function fits are unable to capture this behaviour, under-fitting the high-mass end in all simulations, and showing minimal dependence of $\mathcal{M}^{*}$ and $\alpha$ on halo mass. Varying the rate of the high-mass decline via the $\beta$ parameter in equation (6) cannot eliminate this discrepancy.

A double Schechter function is required in order to fit a low-mass upturn in the L-GALAXIES SMF for $\lg \mathcal{M}_{*} \lesssim 9.5$ : single Schechter fits are too shallow at the low-mass end. None of the IllustrisTNG SMFs show significant evidence of a lowmass upturn, but are very poorly-fit at the high-mass end, particularly for blue satellites. The red satellite SMFs are roughly consistent between IllustrisTNG and GAMA, whereas blue satellites in IllustrisTNG show a large excess at the high-mass end. It thus appears that IllustrisTNG under-estimates the quenching of massive satellite galaxies in group environments. The EAGLE satellite SMF is consistent with IllustrisTNG but limited to lower masses, $\lg \mathcal{M}_{*} \lesssim 11$.

\subsection{Evolution in group environments}

In order to study the effect of group environment on LF/SMF evolution, we compare in Fig. 10 to the (environment-independent) field, after renormalizing the field galaxy numbers to the number of grouped galaxies of particular type and environment. We find that, with the exception of red galaxies, faint and low-mass galaxies are relatively less abundant in group environments at low-redshift, $z<0.1$. Conversely, luminous and massive galaxies, mostly seen at redshifts $z>0.1$, are relatively more common in group environments. The dominant evolutionary effect in group environments is an increasing red fraction with decreasing redshift, relative to the field. The fact that this is seen at redshifts $z<0.3$ suggests that environment quenching of galaxies in groups is an ongoing process.

\subsection{Comparison with previous results}

The overall trend of finding more luminous and massive galaxies in higher-mass haloes can be understood in the context of the hierarchical model of galaxy formation. In this model, massive galaxies accrete much of their stellar mass from sub-haloes, via major 
and minor mergers (e.g. White \& Rees 1978; Cole et al. 2000). Analysing the Illustris simulation, Rodriguez-Gomez et al. (2016) find that while the fraction of stellar mass contributed by accreted stars is only about 10 per cent for Milky Way-sized galaxies, it can be more than 80 per cent for $\mathcal{M}_{*} \sim 10^{12} \mathcal{M}_{\odot}(h=0.7)$ galaxies. It does not automatically follow, however, that the richer environments of massive groups will lead to a higher merger rate, and hence more massive and luminous galaxies. In fact, mergers are expected to be less frequent in high-mass haloes due to the large relative galaxy velocities in these environments (Ostriker 1980; Binney \& Tremaine 1987). However, Sheen et al. (2012) have found that 38 per cent of early-type galaxies in four massive galaxy clusters show evidence of strong merger features (tidal tails, shells, etc.), comparable to what is found in low-density field environments (van Dokkum 2005). Oh et al. (2018) find that 20 per cent of galaxies observed in rich clusters show post-merger signatures, whereas only 4 per cent show evidence of ongoing mergers, in agreement with Sheen et al. (2012). Oh et al. (2018) suggest that the mergers took place before galaxy accretion into the cluster environment, a claim supported by the numerical simulations of Yi et al. (2013).

The increasing characteristic luminosity and stellar mass of galaxies with the mass of their host dark matter halo thus suggests that mergers have been most common in the past history of galaxies accreted into massive haloes. Tomczak et al. (2017), using a semi-empirical model of SMF evolution, show that the majority of galaxies in high-density regions at redshift $z \approx 0.8$ are formed from mergers. We have attempted to extend their model to $z \approx 0.2$, appropriate for the GAMA data, but find that the evolved SMF, even at masses $\lg \mathcal{M}_{*} \sim 10$, is very sensitive to the low-mass cutoff chosen for the initial SMF power law. We therefore prefer to compare our results with more detailed models and simulations such as L-GALAXIES, EAGLE and IllustrisTNG, finding broad agreement in the halo-mass dependence of more massive galaxies.

Galaxies built from multiple mergers are more likely to be spheroidal in morphology (Rodriguez-Gomez et al. 2016). Moreover, Rodriguez-Gomez et al. (2017) find that mergers play an important role in determining galaxy morphology in massive $\left(\mathcal{M}_{*} \gtrsim 10^{11} M_{\odot}\right)$ galaxies in the Illustris simulation, with gaspoor mergers promoting the formation of spheroidal galaxies. In support of this, Man et al. (2016) and Mundy et al. (2017) estimate from observations that about one third of the stellar mass in massive galaxies is acquired via major mergers since redshift $z \approx 3.5$, This merger-driven scenario naturally explains the domination of the bright and high-mass ends of the group LF and SMF by spheroidal galaxies. One should, however, bear in mind that other mechanisms are also likely to come into play in the formation of spheroids, such as 'inside-out' quenching (Tacchella et al. 2018).

Unlike Yang et al. (2008, 2009), we find no evidence of a systematic steepening with halo mass of faint-end/low-mass LF/SMF; in fact the lowest-mass $\mathcal{M} 1$ haloes tend to have the steepest slopes. We caution that apparent trends of Schechter-like parameters should be treated with caution, and can be misleading in cases when the fitting function poorly fits the data. One should also bear in mind that galaxies have had longer to interact in low-redshift (mostly low-mass) haloes, and that this is unlikely to be accounted for by our global evolution corrections.

\subsection{Caveats and future prospects}

The high spectroscopic completeness of the GAMA survey, and the minimum group membership requirement $\left(N_{\mathrm{FoF}}>4\right)$, should result in a higher-fidelity group catalogue than the much larger SDSS catalogue of Yang et al. (2007). However, the GAMA groups are by no means perfect. In particular, comparison with mocks (Fig. 1) suggests that low-mass haloes $\left(\lg \mathcal{M}_{h} \lesssim 13.5\right)$ have masses overestimated by $\Delta \lg \mathcal{M}_{h} \approx 0.5 \mathrm{dex}$. This leads to some small systematic errors in the halo-dependent LF and SMF, particularly at the faint/low-mass end (Appendix B).

Because we analyse a flux-limited sample of GAMA groups, we cannot separate the effects of host halo mass and (observed) group membership. A large, volume-limited sample of galaxy groups would enable more reliable conclusions on the effects of group environment to be drawn. Such a sample will be provided by the upcoming Wide Area VISTA Extragalactic Survey (WAVES; Driver et al. 2019).

\section{ACKNOWLEDGEMENTS}

GAMA is a joint European-Australasian project based around a spectroscopic campaign using the Anglo-Australian Telescope. The GAMA input catalogue is based on data taken from the Sloan Digital Sky Survey and the UKIRT Infrared Deep Sky Survey. Complementary imaging of the GAMA regions is being obtained by a number of independent survey programs including GALEX MIS, VST KiDS, VISTA VIKING, WISE, Herschel-ATLAS, GMRT and ASKAP providing UV to radio coverage. GAMA is funded by the STFC (UK), the ARC (Australia), the AAO, and the participating institutions. The GAMA website is http://www.gama-survey.org/.

JAVM was supported by the Mexican National Council for Science and Technology (CONACyT) scholarship scheme, and thanks Peder Norberg for useful discussions and hospitality. JL (ORCID 0000-0001-5290-8940) acknowledges support from the Science and Technology Facilities Council (STFC) (grant number ST/I000976/1). SDR is supported by a STFC studentship.

We acknowledge the Virgo Consortium and the IllustrisTNG team for making their simulation data available. The EAGLE simulations were performed using the DiRAC-2 facility at Durham, managed by the ICC, and the PRACE facility Curie based in France at TGCC, CEA, Bruyères-le-Châtel. This work also used the 2015 public version of the Munich model of galaxy formation and evolution: L-GALAXIES. The source code and a full description of the model are available at https:// lgalaxiespublicrelease.github.io/.

Finally, we thank two anonymous referees for their careful reading of the manuscript and helpful suggestions for improvement.

\section{DATA AVAILABILITY}

The data underlying this article will be shared on reasonable request to the corresponding author. Tabulated LF and SMF results will be made available via the GAMA website http://www. gama-survey.org/.

\section{References}

Alpaslan M., et al., 2015, MNRAS, 451, 3249

Baldry I. K., Balogh M. L., Bower R. G., Glazebrook K., Nichol R. C., Bamford S. P., Budavari T., 2006, MNRAS, 373, 469

Baldry I. K., et al., 2010, MNRAS, 404, 86

Baldry I. K., et al., 2012, MNRAS, 421, 621

Barden M., et al., 2005, ApJ, 635, 959 
Barsanti S., et al., 2018, ApJ, 857, 71

Benson A., Bower R., Frenk C., Lacey C., Baugh C., Cole S., 2003, ApJ, 599, 38

Berlind A. A., Weinberg D. H., 2002, ApJ, 575, 587

Berlind A. A., et al., 2006, ApJS, 167, 1

Bertin E., Arnouts S., 1996, A\&AS, 117, 393

Binney J., Tremaine S., 1987, Galactic dynamics. Princeton University Press, Prineton, NJ

Blanton M. R., Roweis S., 2007, AJ, 133, 734

Blanton M. R., et al., 2003, AJ, 125, 2348

Bower R. G., Benson A. J., Malbon R., Helly J. C., Frenk C. S., Baugh C. M., Cole S., Lacey C. G., 2006, MNRAS, 370, 645

Boylan-Kolchin M., Springel V., White S. D. M., Jenkins A., Lemson G., 2009, MNRAS, 398, 1150

Brown M. J. I., et al., 2008, ApJ, 682, 937

Cole S., 2011, MNRAS, 416, 739

Cole S., Lacey C. G., Baugh C. M., Frenk C. S., 2000, MNRAS, 319, 168

Colless M., et al., 2001, MNRAS, 328, 1039

Crain R. A., et al., 2015, MNRAS, 450, 1937

Croton D. J., et al., 2005, MNRAS, 356, 1155

Davies L. J., et al., 2019, MNRAS, 483, 5444

Driver S. P., et al., 2009, Astron. Geophys., 50, 5.12

Driver S. P., et al., 2011, MNRAS, 413, 971

Driver S., et al., 2016, MNRAS, 455, 3911

Driver S. P., et al., 2019, The Messenger, 175, 46

Eke V. R., et al., 2004a, MNRAS, 348, 866

Eke V. R., et al., 2004b, MNRAS, 355, 769

Eke V. R., Baugh C. M., Cole S., Frenk C. S., Navarro J. F., 2006, MNRAS, 370,1147

Gonzalez-Perez V., Lacey C. G., Baugh C. M., Lagos C. D. P., Helly J., Campbell D. J. R., Mitchell P. D., 2014, MNRAS, 439, 264

Guo Q., et al., 2014, MNRAS, 442, 2253

Han J., et al., 2015, MNRAS, 446, 1356

Henriques B. M. B., White S. D. M., Thomas P. A., Angulo R., Guo Q., Lemson G., Springel V., Overzier R., 2015, MNRAS, 451, 2663

Hoyle F., Rojas R. R., Vogeley M. S., Brinkmann J., 2005, ApJ, 620, 618

Huertas-Company M., et al., 2015, ApJS, 221, 8

Humason M. L., Mayall N. U., Sandage A. R., 1956, AJ, 61, 97

Kelvin L. S., et al., 2012, MNRAS, 421, 1007

Kelvin L. S., et al., 2014a, MNRAS, 439, 1245

Kelvin L. S., et al., 2014b, MNRAS, 444, 1647

Knobel C., Lilly S. J., Woo J., Kovač K., 2015, ApJ, 800

Lacey C. G., et al., 2016, MNRAS, 462, 3854

Lagos C. d. P., Tobar R. J., Robotham A. S. G., Obreschkow D., Mitchell P. D., Power C., Elahi P. J., 2018, MNRAS, 481, 3573

Lin H., Yee H. K. C., Carlberg R. G., Morris S. L., Sawicki M., Patton D. R., Wirth G., Shepherd C. W., 1999, ApJ, 518, 533

Liske J., et al., 2015, MNRAS, 452, 2087

Loveday J., et al., 2012, MNRAS, 420, 1239

Loveday J., et al., 2015, MNRAS, 451, 1540

Man A. W. S., Zirm A. W., Toft S., 2016, ApJ, 830, 89

Marinacci F., et al., 2018, MNRAS, 480, 5113

McAlpine S., et al., 2016, Astron. Comput., 15, 72

McNaught-Roberts T., et al., 2014, MNRAS, 445, 2125

Merchán M., Zandivarez A., 2002, MNRAS, 335, 216

Merchán M. E., Zandivarez A., 2005, ApJ, 630, 759

Moffett A. J., et al., 2016, MNRAS, 457, 1308

Mortlock A., et al., 2015, MNRAS, 447, 2

Mundy C. J., Conselice C. J., Duncan K. J., Almaini O., Häuler B., Hartley W. G., 2017, MNRAS, 470, 3507

Muñoz-Cuartas J. C., Müller V., 2012, MNRAS, 423, 1583

Naiman J. P., et al., 2018, MNRAS, 477, 1206

Nelson D., et al., 2018, MNRAS, 475, 624

Nelson D., et al., 2019, Comput. Astrophys. Cosmol., 6, 2

Oh S., et al., 2018, ApJS, 237, 14

Ostriker J. P., 1980, Comments Astrophys., 8, 177

Park C., Choi Y., Vogeley M. S., Gott III J. R., Blanton M. R., 2007, ApJ, 658,898
Peng Y.-j., et al., 2010, ApJ, 721, 193

Phillipps S., Driver S. P., Couch W. J., Smith R. M., 1998, ApJ, 498, L119

Phleps S., Wolf C., Peacock J. A., Meisenheimer K., van Kampen E., 2007, A\&A, 468, 113

Pillepich A., et al., 2018, MNRAS, 475, 648

Press W. H., Schechter P., 1974, ApJ, 187, 425

Reddick R. M., Wechsler R. H., Tinker J. L., Behroozi P. S., 2013, ApJ, 771,30

Robotham A., Wallace C., Phillipps S., De Propris R., 2006, ApJ, 652, 1077

Robotham A., Phillipps S., De Propris R., 2010, MNRAS, 403, 1812

Robotham A. S. G., et al., 2011, MNRAS, 416, 2640

Rodriguez-Gomez V., et al., 2016, MNRAS, 458, 2371

Rodriguez-Gomez V., et al., 2017, MNRAS, 467, 3083

Schaye J., et al., 2015, MNRAS, 446, 521

Schechter P., 1976, ApJ, 203, 297

Schlegel D. J., Finkbeiner D. P., Davis M., 1998, ApJ, 500, 525

Sérsic J. L., 1963, Bol. la Asoc. Argentina Astron., 6, 41

Sheen Y.-K., Yi S. K., Ree C. H., Lee J., 2012, ApJS, 202, 8

Springel V., et al., 2005, Nature, 435, 629

Springel V., et al., 2018, MNRAS, 475, 676

Tacchella S., et al., 2018, ApJ, 859, 56

Taylor E. N., et al., 2011, MNRAS, 418, 1587

Taylor E. N., et al., 2015, MNRAS, 446, 2144

Tomczak A. R., et al., 2017, MNRAS, 472, 3512

Viola M., et al., 2015, MNRAS, 452, 3529

Wang L., et al., 2018, A\&A, 618, A1

Wechsler R. H., Tinker J. L., 2018, Annu. Rev. Astron. Astrophys., 56, 435

Weinmann S. M., van den Bosch F. C., Yang X., Mo H. J., 2006, MNRAS, 366, 2

White S. D. M., Rees M. J., 1978, MNRAS, 183, 341

Wright A. H., et al., 2016, MNRAS, 460, 765

Wright A. H., et al., 2017, MNRAS, 470, 283

Xia L., Zhou X., Yang Y., Ma J., Jiang Z., 2006, ApJ, 652, 249

Yang X., Mo H. J., van den Bosch F. C., 2003, MNRAS, 339, 1057

Yang X., Mo H. J., van den Bosch F. C., Jing Y. P., 2005, MNRAS, 356, 1293

Yang X., Mo H. J., van den Bosch F. C., Pasquali A., Li C., Barden M., 2007, ApJ, 671, 153

Yang X., Mo H. J., van den Bosch F. C., 2008, ApJ, 676, 248

Yang X., Mo H. J., van den Bosch F. C., 2009, ApJ, 695, 900

Yi S. K., Lee J., Jung I., Ji I., Sheen Y.-K., 2013, A\&A, 554, A122

York D. G., et al., 2000, AJ, 120, 1579

Zandivarez A., Martínez H. J., 2011, MNRAS, 415, 2553

Zandivarez A., Martínez H. J., Merchán M. E., 2006, ApJ, 650, 137

da Cunha E., Charlot S., 2011, Astrophys. Source Code Libr. Rec. ascl1106.010

da Cunha E., Charlot S., Elbaz D., 2008, MNRAS, 388, 1595

van Dokkum P. G., 2005, AJ, 130, 2647

\section{APPENDIX A: COMPARISON OF FIELD LFS AND SMFS}

We here compare the field (environment-independent) LFs and SMFs for the GAMA data, the FoF mocks, the L-GALAXIES SAM, and two hydrodynamical simulations.

\section{A1 GAMA, mock and TNG $r$-band field LFs}

We compare the $r$-band field LFs from GAMA, FoF mocks and IllustrisTNG in Fig. A1. For GAMA, we use a density-corrected $V_{\max }$ estimator (Loveday et al. 2015) with errors determined from jackknife sampling. Mock LFs are estimated using a standard $V_{\max }$ estimator and errors show the standard deviation between the nine mocks. We see that the brightest galaxies in the mocks are not as bright as those in the GAMA data. Fitting a double Schechter function (equation 6 of Baldry et al. 2012) to the binned LFs, 


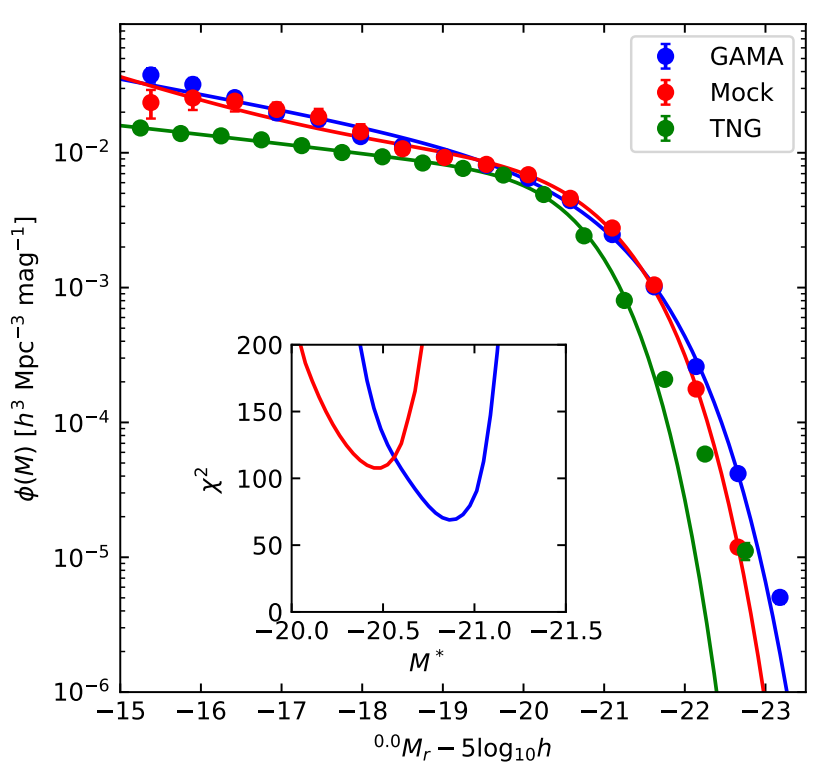

Figure A1. Field galaxy $r$-band LFs for GAMA, FoF mock and TNG. Symbols show $1 / V_{\max }$ estimates (density-corrected for GAMA), and lines show double Schechter function fits. The inset shows GAMA and mock $\chi^{2}$ profiles for characteristic magnitude $M^{*}$; in each case there are 12 degrees of freedom.

we find that the characteristic magnitude $M^{*}$ of GAMA galaxies is about 0.5 mag brighter than in the mocks. The GAMA binned LF faint-end slope is also slightly steeper, although note that the Millennium-based mock catalogues are not expected to be fully complete fainter than $M_{r} \approx-17$ mag. These differences between the GAMA and mock LFs should be borne in mind when comparing group LF results. In particular, one should not focus on differences between GAMA and mocks in any given halo mass bin, but instead compare the trends with halo mass for the real and mock data.

The TNG LF is obtained using the $z=0.2$, TNG300-1 synthetic stellar photometry catalogue, which uses dust model $\mathrm{C}$ from Nelson et al. (2018). We see that while TNG gives a good match to GAMA around the characteristic magnitude $\left(M_{r}-5 \log h \approx-20\right)$ and at the extreme bright end $\left(M_{r}-5 \log h \approx-23\right)$, it predicts far too few faint and moderately bright galaxies. We also note that even a double Schechter function is unable to match the shape of the IllustrisTNG LF at the bright end.

\section{A2 GAMA versus simulated field SMFs}

We compare the field SMFs from GAMA data, the L-GALAXIES SAM, and the EAGLE and IllustrisTNG hydrodynamical simulations in Fig. A2. We see that our estimate of the GAMA field galaxy SMF agrees well with previous GAMA estimates by Baldry et al. (2012) and Wright et al. (2017, their double Schechter function fit). The simulations agree well with the GAMA observations, except that IllustrisTNG over-predicts the numbers of very massive $\left(\lg \mathcal{M}_{*} \gtrsim 11\right)$ galaxies. This over-abundance of simulated, very massive galaxies suggests that the effects of AGN feedback in IllustrisTNG may be underestimated in such hosts. This would also at least partly explain the IllustrisTNG high-mass excess seen in group environments (Fig. 8).

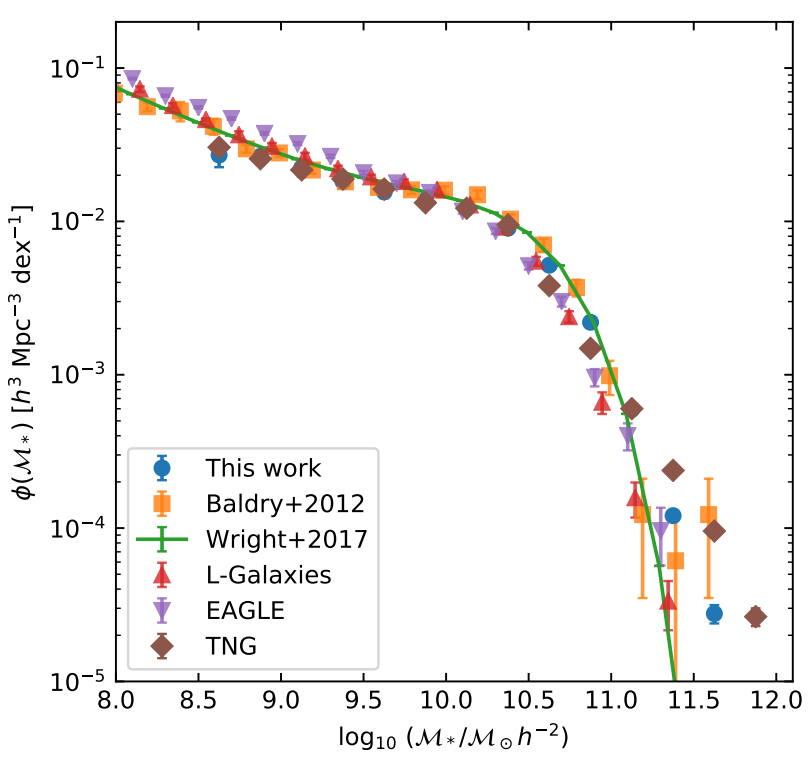

Figure A2. Field galaxy SMFs for GAMA estimated by the present work and two previous GAMA papers, along with predictions from the L-GALAXIES SAM and the EAGLE and IllustrisTNG hydrodynamical simulations.

\section{APPENDIX B: FOF VERSUS HALO MOCK LF RESULTS}

We compare group LF results obtained using the FoF and halo mocks in Fig. B1. Note that in this comparison, the same mock galaxies have been used. The only difference lies in how the galaxies are assigned to groups, and how the masses of the groups are determined.

The composite (central plus satellite) halo and FoF nonparametric LF estimates are significantly different (exceeding 95 per cent confidence) for all but the lowest mass bin. We see that the effect of using FoF group-finding and luminosity-based masses is to change the best-fitting Schechter parameters for the satellite galaxies by around $1-3 \sigma$. The central galaxy LFs appear to be consistent in peak luminosity apart from in FoF $\mathcal{M} 1$ haloes, where they are fainter.

FoF mocks tend to have slightly less steep faint-end slopes than halo mocks; all are significantly under-fitting the faint end, particularly in $\mathcal{M} 3$ groups. In the lowest-mass haloes, $\mathcal{M} 1$, FoF mocks have fainter characteristic magnitudes $M^{*}$; in all other haloes, there is no significant difference.

Insofar as the mock catalogues are representative of the GAMA data, we can infer that the GAMA LF Schechter parameters are likely to be biased by $\sim 1 \sigma$ in intermediate mass bins, with slightly worse errors in the lowest- and highest-mass haloes. When comparing GAMA data with mocks, we use the FoF mocks, under the assumption that they suffer similar biases to the GAMA groups.

\section{APPENDIX C: TESTING LF AND CLF ESTIMATORS}

In this appendix we compare estimates of the LF and CLF from simple simulations of known CLF, showing that the LF may be recovered without bias, but that an unbiased CLF estimate is only possible from a volume-limited sample. We first describe our CLF estimator (our LF estimator is described in Section 3.1), and then describe the generation of simple group and galaxy catalogues with 


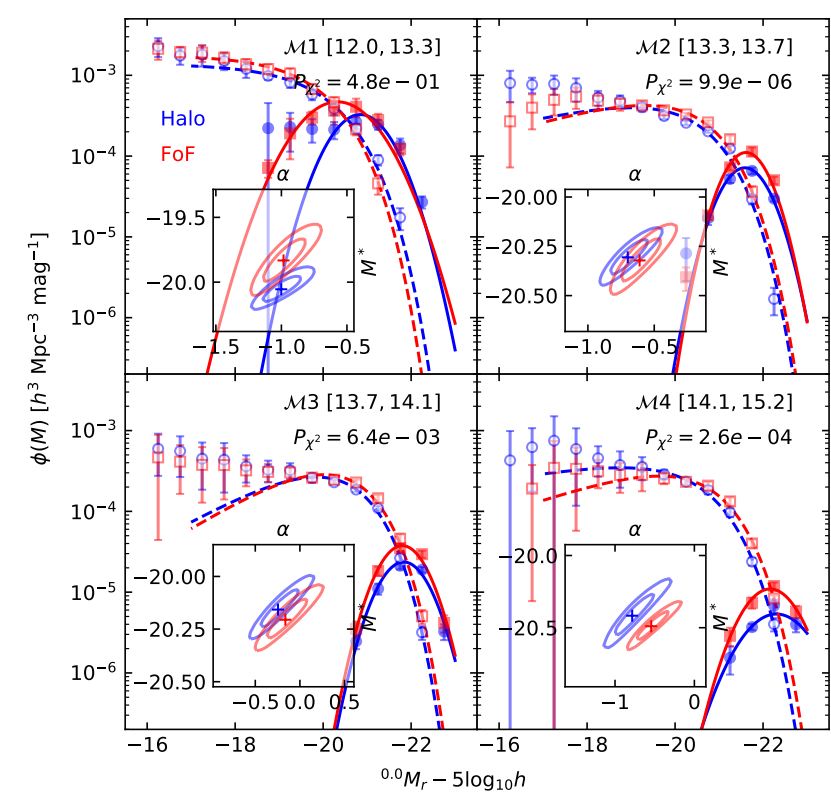

Figure B1. Halo mass dependent LFs for halo (blue circles) and FoF (red squares) mocks. Filled markers and continuous lines show central galaxy LFs with their best-fitting log-normal function. Open markers and dashed lines show satellite galaxy LFs with their best-fitting Schechter function. Insets show 1- and 2- $\sigma$ likelihood contours on the Schechter parameters $\alpha$ and $M^{*}$. Also shown in each panel is the $\chi^{2}$ probability that the composite (central plus satellite) non-parametric LFs follow the same distribution. The difference is statistically significant for all mass bins apart from $\mathcal{M} 1$.

known CLF. Finally, we present and discuss the recovered CLFs and LFs from these simulations.

\section{C1 CLF estimator}

To estimate CLFs for a given bin of halo mass, we consider the member galaxies of the groups in that mass bin. The CLF is given by the absolute magnitude histogram of the member galaxies, weighting each galaxy by the reciprocal of the number of groups in the halo mass bin in which the galaxy could in principle be observed. In other words, for galaxy $i$ which would be visible to redshift $z_{\lim , i}$, we count groups that lie in the redshift range $\left[z_{\mathrm{lo}}, \min \left(z_{\mathrm{hi}}, z_{\mathrm{lim}, i}\right)\right]$, where $\left(z_{\mathrm{lo}}, z_{\mathrm{hi}}\right)$ are the sample redshift limits. This is equivalent to the 'direct matching' method of Guo et al. (2014, equation 4), except that we normalize the CLF on a per-galaxy basis, rather than on a per-magnitude bin or mass bin basis, thus allowing account to be taken of individual galaxy $K$ corrections.

Note that by normalising the galaxy counts by the number of groups in which each galaxy could be seen, one automatically corrects for radial density variations, whether due to large-scale structure or number density evolution, assuming that group counts vary in the same way as galaxy counts, and so no explicit corrections for $\Delta(z)$ and $P(z)$ are needed. One would still apply luminosity evolution corrections for a sample in which luminosity is evolving.

\section{C2 Simulated group and galaxy catalogues}

50,000 group masses are chosen at random over the range $\lg \mathcal{M}_{h}=$ $[12,15]$ from a Schechter mass function with somewhat arbitrary,

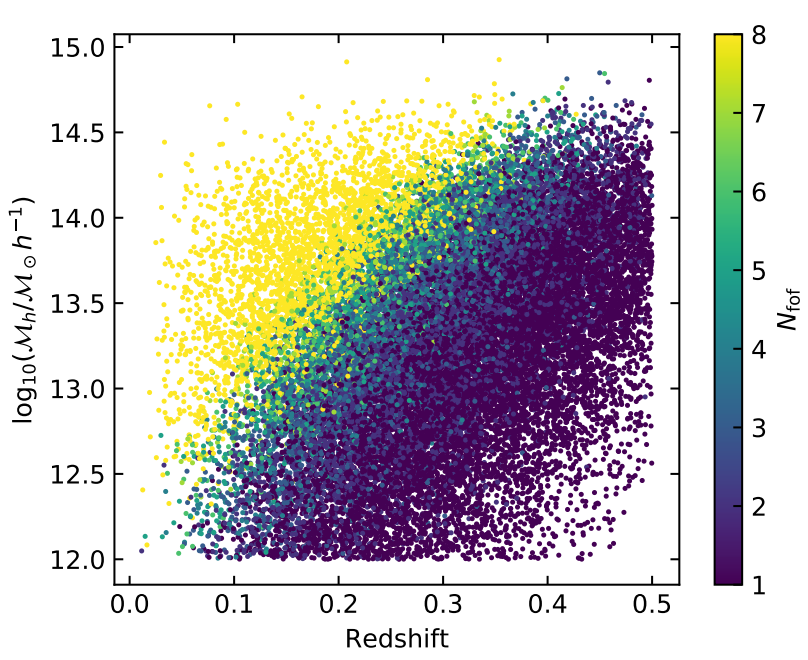

Figure C1. Halo mass-redshift distribution of around 22,000 simulated groups chosen at random from those with at least one visible member galaxy. Colour-coding indicates the number of visible group members, with yellow corresponding to eight or more members.

but not unreasonable, shape parameters $\alpha_{\mathcal{M}}=-1, \lg \mathcal{M}_{h}^{*}=14$. Each group is assigned a redshift randomly drawn from a distribution that is uniform in comoving volume over the redshift range $z=[0.002,0.5]$. These group masses and redshifts are written out to a simulated group catalogue.

Within each group we generate galaxies with luminosities drawn at random over the absolute magnitude range $M=$ $[-24,-15]$ mag from Schechter functions whose parameters vary with group mass $\lg \mathcal{M}_{h}$ as follows:

$$
\begin{aligned}
\alpha & =-1.4-0.2 \Delta \mathcal{M}, \\
M^{*} & =-21.0-0.5 \Delta \mathcal{M}, \\
\lg \phi^{*} & =1.0+0.5 \Delta \mathcal{M},
\end{aligned}
$$

and where $\Delta \mathcal{M}=\lg \mathcal{M}_{h}-\lg \mathcal{M}_{h}^{*}$. These CLF parameters are chosen to roughly match the satellite CLFs of Yang et al. (2008). The number of galaxies generated in each group is chosen at random from a Poisson distribution whose mean is given by integrating the group's CLF over the magnitude range $M=[-24,-15]$ mag. Galaxies are assigned the same redshift as their host group, and apparent magnitudes are calculated using the same $K$-corrections as the GAMA mock catalogues (R11, equation 8), but with no evolution. We write out a simulated galaxy catalogue containing those galaxies with apparent magnitude $m<19.8 \mathrm{mag}$. On average, about 2730 groups in each simulation contain five or more visible member galaxies, comparable with the number of GAMA groups in our observed sample.

Altogether, nine simulated group and random catalogues are generated. While these simulated catalogues do not attempt to model imperfections in group finding or mass estimation, they do allow us to investigate any biases in the recovered LFs or CLFs caused by sample selection effects, particularly those associated with requiring observed groups to have a minimum galaxy membership.

Fig. C1 plots the halo mass-redshift distribution for our simulated groups that contain at least one visible galaxy. It is clear that low mass groups are incomplete at high redshift, even when only a single observed $(m<19.8 \mathrm{mag})$ member galaxy is required. With a 


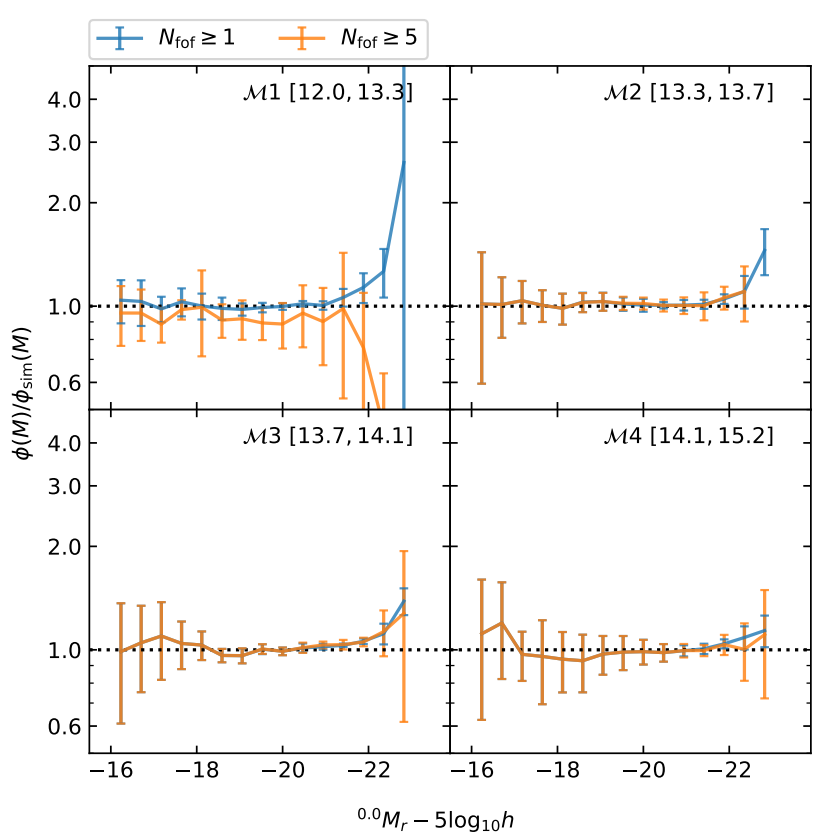

Figure C2. Ratio of recovered to input LF from simulated catalogues. Blue and orange error bars, representing the RMS scatter between simulations, show LF ratios for groups with at least one and five detected members, respectively.

membership threshold of 5 galaxies (green and yellow points), this incompleteness extends to all group masses. Redshift incompleteness is difficult to quantify in an observed sample, due to significant scatter in the relation between halo mass and $N$ th brightest galaxy luminosity. The effects of group redshift incompleteness on the recovered LF and CLF are explored in the next section.

\section{C3 Recovered LFs and CLFs}

LFs and CLFs are determined from our simulated catalogues in the same way as for the GAMA mocks. The ratios of the recovered to simulated LFs and CLFs, (evaluated individually for each group, and then summed within mass bins), are shown in Figs. C2 and C3 respectively. For the LF ratio plots, we have rescaled the input (per group) CLFs to ( $1 / V_{\max }$-weighted) LFs by the factor $N_{\text {group }} / V$, where $N_{\text {group }}$ is the total number of groups simulated in each mass bin, and $V \approx 3.6 \times 10^{7} h^{-3} \mathrm{Mpc}^{3}$ is the effective volume of the simulations, assuming that they cover the same sky area as the GAMA mocks. It is not possible to scale observed LFs and CLFs in this way, since we do not know the total number of groups in each mass bin, only the number that are observed.

The ( $V_{\max }$-normalized) LFs (Fig. C2) are recovered with nearzero bias, albeit with large scatter in low-mass groups, even when a minimum group membership of five galaxies is imposed. By lowering the group membership threshold, we are able to constrain the LF to brighter magnitudes, with little improvement to the faint-end estimates.

The recovered (group-normalized) CLFs (Fig. C3) only do a good job in matching the simulation input when all simulated groups (blue lines), including even those that contain no visible galaxies, are included in the normalisation of the CLF. By including only groups with one or more observed members (orange lines),

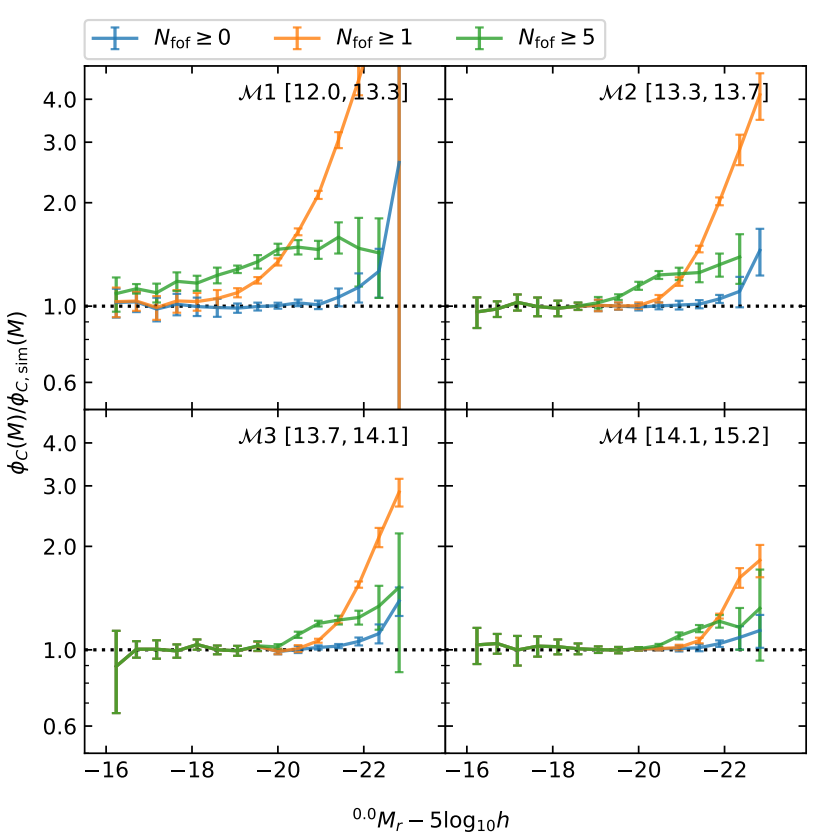

Figure C3. Ratio of recovered to input CLF from simulated catalogues. Blue, orange, and green error bars show CLF ratios for groups with at least zero, one and five detected members, respectively.

the bright end of the CLF is overestimated, particularly in low-mass groups. This overestimation extends to fainter magnitudes as group membership cut is increased to five or more galaxies, although interestingly is then less severe at brighter magnitudes. Overestimation of the CLF occurs because luminous galaxies are visible to high redshift, at which the group sample is increasingly incomplete with decreasing mass and increasing membership threshold (Fig. C1). Since there is a wide scatter in the correlation of halo mass with $N$ th brightest galaxy luminosity, redshift incompleteness is difficult to quantify. For observed group catalogues, one only knows of those groups that have at the very least one member, and so without modelling of the halo mass function, one cannot calculate a reliable CLF without imposing stringent redshift limits on the sample.

We have re-evaluated the CLFs for groups with five or more members applying redshift cuts of $z<0.1$ and $z<0.2$ (Fig. C4). For these simulations, but not necessarily for GAMA groups, we see that a redshift cut of $z<0.1$ enables a reliable CLF estimate for all but the lowest-mass groups. A less stringent cut of $z<0.2$ gives acceptable results for mass bins $\mathcal{M} 3$ and $\mathcal{M} 4$.

Having established that we can recover $V_{\max }$-normalized LFs without bias for all group masses without any redshift cuts, we investigate the effects of redshift selection on the recovered $L F$ in Fig. C5. For higher-mass groups, $\mathcal{M} 3$ and $\mathcal{M} 4$, the LFs in all three redshift ranges are recovered without bias. For mass bin $\mathcal{M} 2$, the LFs in the highest-redshift range, $0.2<z<0.3$, are biased low. For the lowest-mass groups, $\mathcal{M} 1$, the intermediate redshift range, $0.1<z<0.2$, is biased low; there are too few galaxies at higher redshifts to measure an LF at all. We conclude that it should be possible to constrain LF evolution in $\lg \mathcal{M}_{h} \gtrsim 13.7$ groups out to redshift $z \approx 0.3$. For groups in mass bin $\mathcal{M} 2$, evolution can be reliably constrained to $z \approx 0.2$. No determination of LF evolution is possible for groups in the lowest mass bin. 


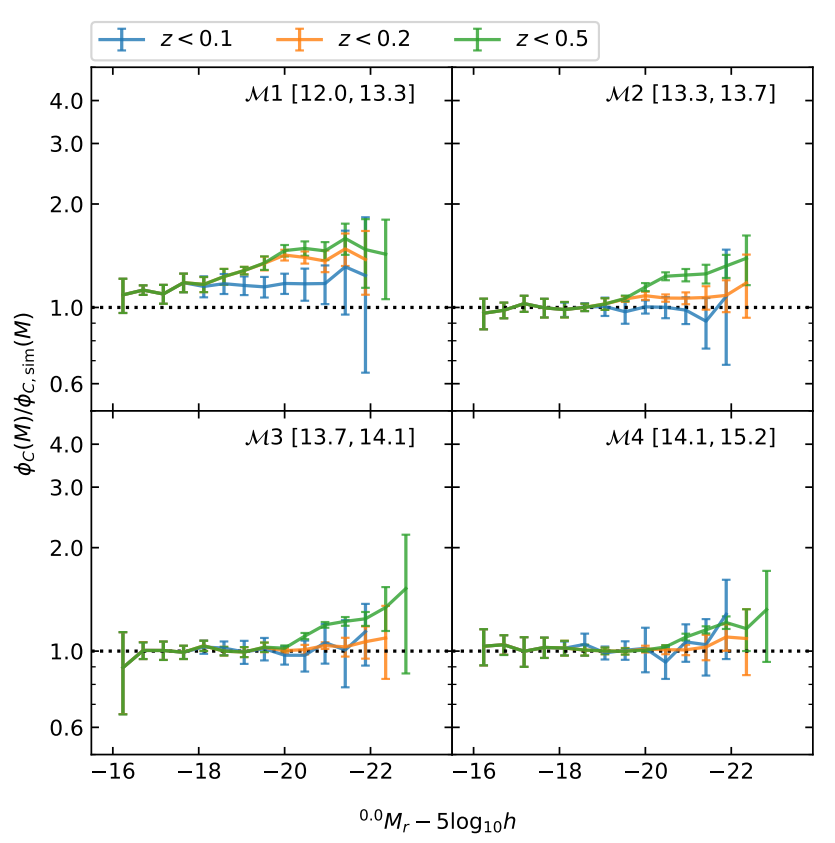

Figure C4. Ratio of recovered to input CLF from simulated catalogues for groups with five or more members. Blue and orange error bars show CLF ratios after applying redshift cuts of 0.1 and 0.2 respectively. The green error bars are the same as in Fig. C3.

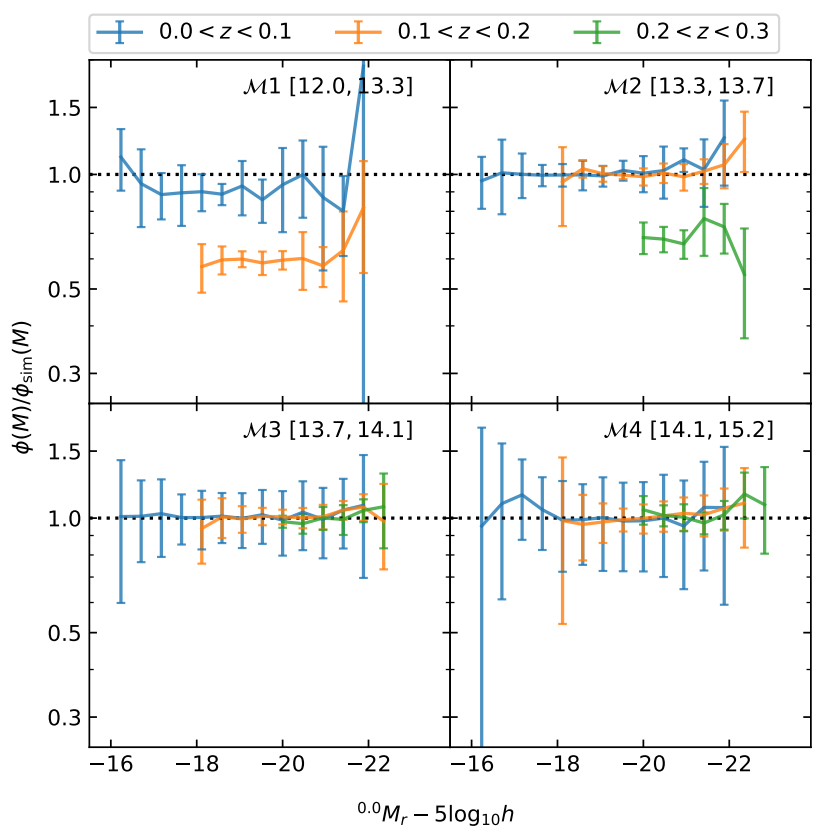

Figure C5. Ratio of recovered to input LF from simulated catalogues measured in slices of redshift. Blue, orange, and green error bars show LF ratios for groups with five or more members at redshift $0.0<z<0.1$, $0.1<z<0.2$, and $0.2<z<0.3$, respectively.
In this appendix, we have demonstrated that the $V_{\max }{ }^{-}$ normalized LFs may be recovered from GAMA-like data without bias, even when one is restricted to groups with five or more members, with a significant incompleteness in mass-redshift space, as seen in Fig. C1. CLFs may be recovered without bias only when one normalizes by the total number of groups in the relevant mass and redshift range, including those with no visible galaxy members (clearly impossible for an observed, flux-limited sample), or by applying stringent redshift cuts to obtain a sample that is volumelimited in group mass (the approach taken by Yang et al. 2008, 2009). For that reason, we present $V_{\max }$-normalized LFs and SMFs rather than group-normalized CLFs and CSMFs in this paper.

This paper has been typeset from a $\mathrm{T}_{\mathrm{E}} \mathrm{X} / \mathrm{LT}_{\mathrm{E}} \mathrm{X}$ file prepared by the author. 